Portland State University

PDXScholar

\title{
Near-Field Acousto Monitoring Shear Interactions Inside a Drop of Fluid: The Role of the Zero-Slip condition
}

Xiaohua Wang

Portland State University

Rodolfo Fernandez Rodriguez

Portland State University, rodolfo.f.r@gmail.com

Nan Li

Bronx Science High School

Hsien-Chih Hung

Portland State University

Anuradha Venkataraman

Portland State University, maverick.nicenwise@gmail.com

Follow this and additional works at: https://pdxscholar.library.pdx.edu/phy_fac

Part of the Fluid Dynamics Commons

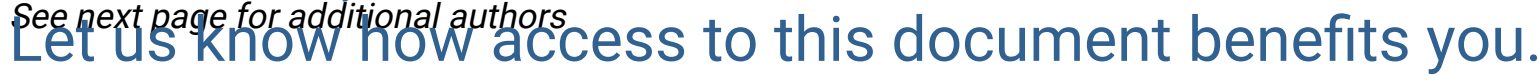

\section{Citation Details}

Wang, Xiaohua; Fernandez Rodriguez, Rodolfo; Li, Nan; Hung, Hsien-Chih; Venkataraman, Anuradha; Nordstrom, Richard; and La Rosa, Andres H., "Near-Field Acousto Monitoring Shear Interactions Inside a Drop of Fluid: The Role of the Zero-Slip condition" (2016). Physics Faculty Publications and Presentations. 251.

https://pdxscholar.library.pdx.edu/phy_fac/251

This Post-Print is brought to you for free and open access. It has been accepted for inclusion in Physics Faculty Publications and Presentations by an authorized administrator of PDXScholar. Please contact us if we can make this document more accessible: pdxscholar@pdx.edu. 


\section{Authors}

Xiaohua Wang, Rodolfo Fernandez Rodriguez, Nan Li, Hsien-Chih Hung, Anuradha Venkataraman, Richard Nordstrom, and Andres H. La Rosa 
Xiaohua Wang, ${ }^{1}$ Rodolfo Fernandez, ${ }^{1}$ Nan Li, ${ }^{2}$ Hsien-Chih Hung, ${ }^{1}$ Anuradha Venkataraman, ${ }^{1}$ Richard Nordstrom, ${ }^{1}$ and Andres H. La Rosa ${ }^{1, a)}$

${ }^{1}$ Department of Physics, Portland State University, P.O. Box 751; Portland, Oregon 97207

${ }^{2}$ Bronx Science High School, 75 West 205th Street, Bronx, NY 10468

A full understanding of nanometer-range (near-field) interactions between two sliding solid boundaries, with a mesoscopic fluid layer sandwiched in between, remains challenging. In particular, the origin of the blue-shift resonance frequency experienced by a laterally oscillating probe when approaching a substrate is still a matter of controversy. A simpler problem is addressed here, where a laterally oscillating solid probe interacts with a more sizable drop of fluid that rests on a substrate, aiming at identifying interaction mechanisms that could also be present in the near-field interaction case. It is found that the inelastic component of the probe-fluid interaction does not constitute the main energy-dissipation channel and has a weak dependence on fluid's viscosity, which is attributed to the zero-slip hydrodynamic condition. In contrast, the acoustic signal engendered by the fluid has a stronger dependence on the fluid's viscosity (attributed also to the zero-slip hydrodynamic condition) and correlates well with the probe's resonance frequency red-shift. We propose a similar mechanisms happens in near field experiments, but a blue-shift in the probe's resonance results as a consequence of the fluid molecules (subjected to the zero-slip condition at both the probe and substrate boundaries) exerting instead a spring type restoring force on the probe.

\footnotetext{
a) Author to whom correspondence should be addressed. Electronic mail: andres@pdx.edu
} 


\section{Publishing vTRODUCTION}

Quartz tuning forks (QTF) have been successfully incorporated into scanning probe microscopy (SPM). ${ }^{1,2}$ Upon electrical excitation, the piezoelectric property of the QTF allows setting its two tines into lateral oscillations, one of which carries an attached probe (typically few millimeters long, $\sim 100 \mu \mathrm{m}$ wide but tapered to an apex of nanometer-sized radius). A probe approaching a sample (referred here as a substrate with its naturally adsorbed fluid layer ${ }^{3}$ ) experiences near-field "shear-forces" that significantly affect the TF oscillations (near-field refers here to the nanometer probe-substrate separation distance). The perception that an adsorbed fluid layer of few nanometer thickness can exert such a strong effect on a millimeter size probe springs from the fact that, as it is well known, confined mesoscopic fluids display properties quite different than the bulk (namely, enhanced shear viscosity, prolonged relaxation time, confinement-induced phase transformation) ${ }^{4}$ However, the exact nature of the near-field "shear forces" and the involved striking properties of mesoscopic fluids are not yet well understood.

The dynamic behavior of mesoscopic fluids trapped between the boundaries of a probe and substrate is indeed complex. But if we focused instead on the interaction involving a more sizeable volume of fluid (few $\mu \mathrm{L}$ ), the complexity will be reduced considerably, still we may be able to identify a subset of characteristics also present in the mesoscopic-volume near-field case. Herein we describe a systematic implementation of such tests, using probes of various sizes and fluid droplets of various viscosities. What type of responses from the probe and the fluid would we see by inserting the probe to various immersion lengths? What is the role of the fluid in these interactions, and how does the fluid response relates to the probe's physical parameters (shift in resonance frequency for example)? Would a change of the droplet volume affect the results? Upon performing these tests, what responses could we infer if an actual surface were placed closer to the probe? Could these insights be extrapolated to interpret the results from mesoscopic fluid cases? These are the questions addressed herein.

A new feature in the measurements reported here (but no present in a typical QTF-SPM) involves using an acoustic transducer to monitor the droplet fluid response (in addition to the QTF signal that monitors the probe's oscillation amplitude). The combined QTF and acoustic sensing strategy has been called Shear-force Acoustic Near-field Microscopy (SANM). ${ }^{5}$ From 
Publishineg field measurements ${ }^{6,7}$ it is known that typically the acoustic signal strengthens as the QTF signal weakens, however the vertical range of comparison is obviously limited. By using instead a drop of water one has the ability to achieve deeper immersion into the fluid and, thus, could allow making a clearer correlation, if exists, between the QTF and acoustic signals. Possible factors such as damping, mass loading, and energy transfer, will be discussed in the context of synchronous measurements of changes in amplitude, resonance frequency shift, and in response to variations in probe diameter and fluid viscosity.

\section{EXPERIMENTAL DETAILS}

\section{A. Control variables}

Table 1 shows the set of variables investigated to evaluate their influence in the probe-fluid interaction. The primary variables, namely the probe diameter, probe's immersion length, and fluid's viscosity, were chosen as they were a priori estimated to have the best chance to exhibit tangible consequences. The secondary parameters like driving force, fluid volume and water evaporation time were investigated in order to verify the stability and reproducibility of the results.

Each test followed a preparation of the sample surface, adding a water droplet, submersion of the probe into the droplet, and measurement with the SANM system. The hydrophilic character of atomically flat mica allowed an easy spread of the fluid on the surface (still forming droplet-type geometry). The fiber protruding from one of the QTF's tines is dipped into a droplet of glycerol aqueous solution ( $\sim 5 \mu \mathrm{L}$ in volume) placed on a mica disk. The submersion length was controlled using a set of fine-pitch screws (100 TPI precision, $7 \mu \mathrm{m}$ travel per $10^{\circ}$ turn, AJS1002 from Newport), complemented with the nanopositioning stage (Nano-OP65, $65 \mu \mathrm{m}$ range linear motion, $0.13 \mathrm{~nm}$ precision; from Mad City Labs, Inc.) built into the SANM. All experiments reported herein were performed under ambient temperature $\sim 23{ }^{\circ} \mathrm{C}$ and relative humidity of $\sim 45 \%$. 


\begin{tabular}{|c|c|c|}
\hline Figure & Control Variable & Tests series \\
\hline Fig 2 & Depth of probe in water droplet & $\begin{array}{l}\text { Depth }=0^{-}, 0^{+}, 10,20,30,40,80 \text {, } \\
120,160,200,240,280 \mu \mathrm{m}\end{array}$ \\
\hline Fig 3, 4 & Probe diameter & $125,114,98,81 \mu \mathrm{m}$ \\
\hline Fig 5, 6 & Viscosity & $\begin{array}{l}\text { Water droplet of } 0 \%, 30 \%, 40 \% \text {, } \\
50 \% \text { glycerin concentration }\end{array}$ \\
\hline Fig 7 & Driving force dependence & 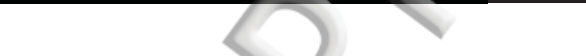 \\
\hline Fig 8 & Water evaporation time & \\
\hline Fig 9 & Water volume. & \\
\hline
\end{tabular}

Table 2. Baseline Parameters

\begin{tabular}{|l|l|}
\hline Probe diameter & $125 \mu \mathrm{m}$ cylindrical, optical glass fiber \\
\hline Probe mounting & Outside one of the QTF prongs \\
\hline Nominal QTF AC voltage excitation & $40 \mathrm{mV} \mathrm{V}_{\text {rms }}$ amplitude \\
\hline Glycerol concentration & $0 \%$ w.t. (i.e., 100\% distilled water) \\
\hline Spectra recording time & $20 \mathrm{~s}$ \\
\hline
\end{tabular}

\section{B. Probe Fabrication}

We use commercial QTF (520-TFC3X8-X, 12.5 pF, from Mouser Electronics) with nominal frequency of $32768 \mathrm{~Hz}$, and with a calculated spring constant $K_{\text {stat }}=(E / 4) w(t / L)^{3}=26 \times 10^{3} \mathrm{~N} / \mathrm{m}$ (the value obtained using the prong's dimensions $L=3.8 \mathrm{~mm}, t=0.6 \mathrm{~mm}$, and $w=0.35 \mathrm{~mm}$, and the quartz elastic modulus $E=7.8710^{10} \mathrm{~N} / \mathrm{m}^{2}$ ). After mounting the probe, the mechanical quality factor $Q$ fall around $10^{3}$. For constructing the complete probe, the QTF is removed from its vacuum lid and a cleaved optical fiber (SMF-28 Corning) of $3 \mathrm{~mm}$ in length and $125 \mu \mathrm{m}$ initial diameter, is glued to one of the QTF prongs; the fiber purposely protrudes $\sim 1 \mathrm{~mm}$ beyond the prong so it can be partially immersed into a drop of liquid. For the purpose of additional tests presented herein, we also prepared glass fibers of reduced diameters through a chemical etching process that uses buffered hydrofluoric acid (BHF) solution. ${ }^{8}$ By using BHF solution with a volume ratio of $\mathrm{NH}_{4} \mathrm{~F}: \mathrm{HF}: \mathrm{H}_{2} \mathrm{O}=2: 1: 1$, the fiber becomes uniformly thinner.

\section{Liquid preparation}

Glycerol-water solutions of different viscosities were prepared by mixing calculated weights of glycerol and distilled water as followed from the literature. ${ }^{9,10}$ For pure water $(0 \%$ glycerin concentration): density $\rho_{0}=997.34 \mathrm{~kg} / \mathrm{m}^{3}$; dynamic viscosity $\gamma_{0}=1.005$ centipoise, where 1 
Publishiceg ipoise $=(\mathrm{cP})=10^{-3} \mathrm{~N} \mathrm{~s} / \mathrm{m}^{2}$. For $50 \%$ glycerin concentration: $\rho_{50}=1129.65 \mathrm{Kg} / \mathrm{m}^{3}$ and $\gamma_{50}=6.000 \mathrm{cp}$.

\section{Description of the Experimental Measurements}

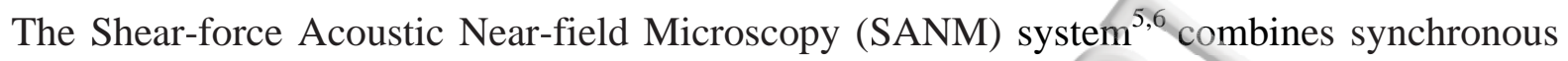
detection of two signals, $i$ ) the electrical QTF signal (the current measured by the lock-in \#1 in Fig. 1) from which one can retrieve the probe's amplitude of oscillation (as described in Results section below), and ii) the acoustic signal (the current from the acoustic transducer monitored by the lock-in \#2) that measures the acoustic signal generated at the liquid droplet. Both are acquired simultaneously while a cleaved optical fiber oscillates laterally and partially-immersed in the fluid. Here the fluid is a droplet of glycerol aqueous mixture $(\sim 5 \mu \mathrm{L})$ placed on a mica disk substrate (which contrasts with a "mesoscale" fluid film involved in near-field measurements.) The configuration of the experimental setup is shown in figure 1.

The most general observed behavior (as will be shown in more detail in the next sections) is a QTF signal (the probe's amplitude of oscillations) decreasing while the acoustic signal (response from the fluid) gaining strength as the probe progressively gets immersed into the bulk liquid. At a given immersion length, both signals are recorded across the frequency spectrum while driving the QTF with a harmonic voltage of constant amplitude. The individual spectra are then analyzed for peak frequency, mechanical quality factor $Q$, and resonance frequency shifts relative to baseline conditions. Since the electrical detection of the probe's amplitude has a drawback in the QTF's inherent capacitance (which modifies the spectral response and, thus, does not reflect an accurate measurement of the QTF's prongs oscillation amplitude), ${ }^{11}$ the spectrum is fit to an RLC equivalent circuit in order to separate out the capacitance contribution and thus calculate more accurately the probe's amplitude of oscillation. ${ }^{12,13}$ This procedure gives a current-to-amplitude calibration factor of $2.5 \mathrm{nA} / \mathrm{nm}^{5}$ 
This

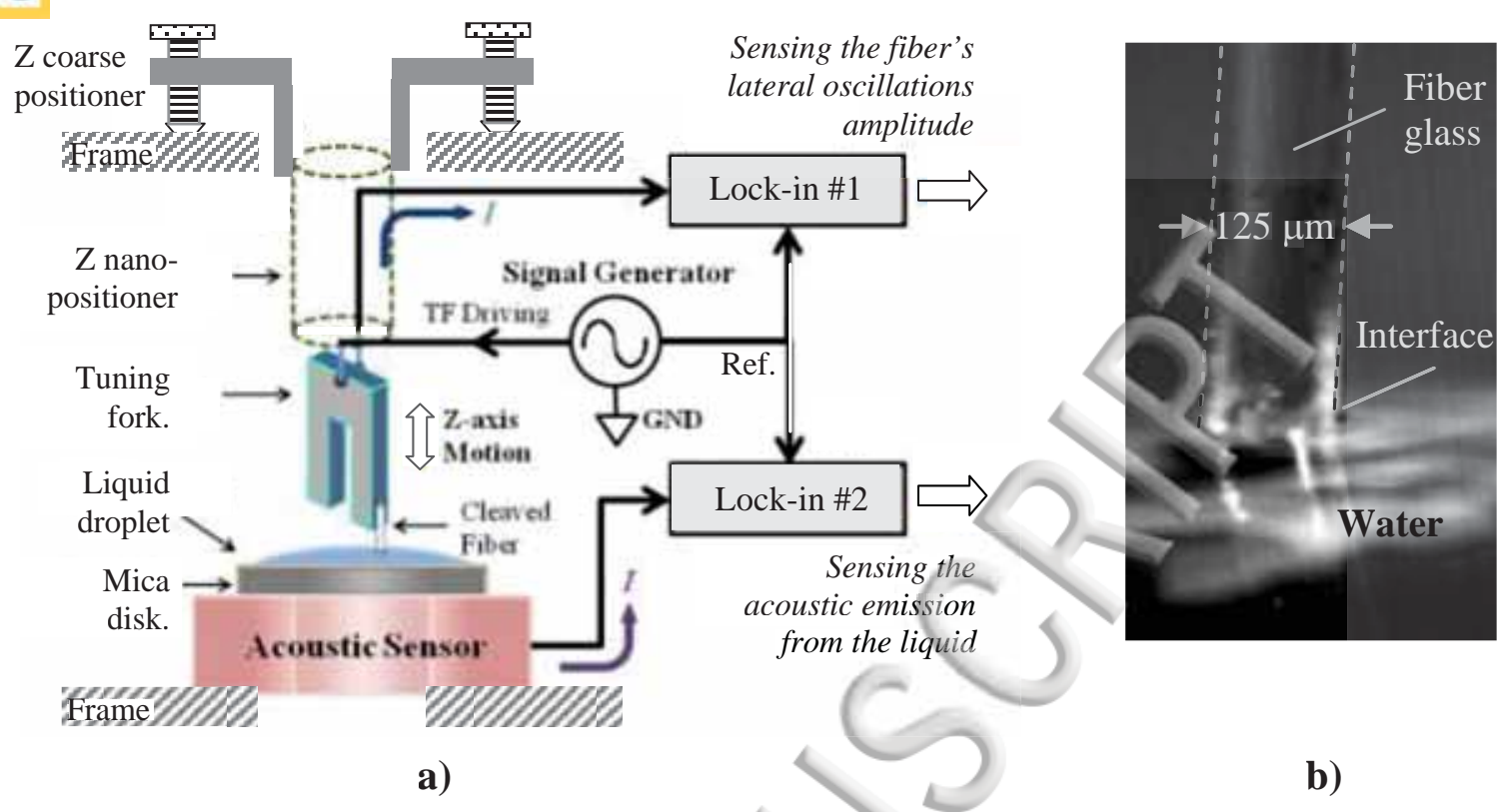

Fig. 1 a) Schematic of the Shear-force Acoustic Near-field Microscope (SANM) setup. b) Optical image of a cleaved fiber probe right after its immersion into the the fluid.

Due to the lateral motion of the probe, an acoustic signal is generated inside the droplet, which couples to the substrate and reaches the acoustic transducer (SE32-Q sensor of $10 \mathrm{~mm}$ diameter sensitive area, and customized for maximum response near $32 \mathrm{kHz}$; from Score Atlanta Inc.) The substrate and the acoustic sensor are in intimate mechanical contact. It is observed that the response from both sensors, the QTF and the acoustic transducer, vary linearly with the amplitude of the ac driving voltage, as described in more detailed in the Results section below.

\section{The ADDITIONAL INERTIAL MASS MODEL}

\section{A. Simple harmonic oscillator model of the QTF probe}

A tuning fork vibrating with limited or negligible interaction with an external environment is usually well described by analyzing the motion of just one of its prongs as a cantilever beam vibrating in flexure. The eigen-frequencies ${ }^{14,15}$ of such a system are given by $2 \pi f_{n}=\left(\alpha_{n} / L\right)^{2}(E I / \rho A)^{1 / 2}$, where the cantilever dimensions are $\mathrm{T}, \mathrm{W}$, and $\mathrm{L}$ (thickness, width, length of the individual prong), $\mathrm{A}=\mathrm{WT}$ is the individual prong's cross section area, $\mathrm{E}$ and $\rho$ are 
Publishithg elastic constant and density of quartz respectively, and $I$ the areal momentum of inertia. The values of $\alpha_{n}$ are determined by the expression imposed by the boundary condition $\left(\cos \alpha_{n} L\right)\left(\cosh \alpha_{n} L\right)+1=0$.

\section{B. Interaction of the probe with a fluid described in terms of an additional inertial mass}

However the purpose here is not to ignore the environmental surrounding the QTF, but rather to characterize the interaction of the probe with a droplet. It turns out, nonetheless, that the frequency response of an elastic beam immersed in a viscous fluid constitutes a formidable problem. ${ }^{16}$ Even for very simple structures like beams and plates, an analytical solution involves rather complicated functions of the wavelength, frequency and dimensional shape factors. ${ }^{17,18}$ However, given the fact that the experimental results reported below reveal signatures that can be accounted by a simple harmonic motion model, it is justified then to attempt a much simpler description as follows.

When a solid body undergoes oscillatory motion inside a fluid medium, the extra energy needed to keep the fluid in motion can be taken into account by an equivalent "additional inertial mass $\Delta m$ " added to the cantilever oscillations, which has an effect in the value of the probe's resonant frequencies. Assuming that the added inertia is much smaller than the mass of the prong, the modified eigen-frequencies are given by $f_{n}=(1 / 2 \pi)\left(\alpha_{n} / L\right)^{2}(E I /[\rho A+\mu])^{1 / 2}$ $\approx f_{\text {on }}\left(1-\frac{1}{2} \frac{\mu}{\rho A}\right)$, where $f_{\text {on }}$ stands for the eigen-frequencies outside the fluid, and $\mu$ is the added mass inertia per unit length. It turns out that for a body of cylindrical geometry $\mu \approx 2 m_{o} / L$, where $m_{o}$ is the mass of the fluid volume displaced by the QTF prong (the factor 2 in front of this expression is associated to a cylindrical geometry)..$^{17,18}$ This gives $f_{n} \approx f_{\text {on }}\left(1-\frac{m_{o}}{M_{\text {Prong }}}\right)$, where $M_{\text {Prong }}$ is the mass of one of the QTF's prongs.

However, the description above assumes that the prong is fully immersed in the fluid, while in our case only part of the attached probe is immersed. Hence, if we considered a mass $m_{\mathrm{o}}=\Delta m_{\text {fluid }}$ (the mass of the fluid volume displaced by the partially submerged probe) as an added mass whose location is concentrated at the end of the prong, its effect on the change in the cantilever's resonance frequency would be greater compared to a similar mass distributed over 
Publishithg full length of the QTF prong. On the other hand, it has been pointed out in the literature that, when describing the dynamics of a QTF, the coupling between the two prongs should also to be taken into account; ${ }^{19}$ so a mass greater than the mass of a single prong $M_{\text {Prong }}$ should be considered, whose effect would be to lower the resonance frequency value. Thus, the influence of these two factors on the resonance frequency tend to cancel each other; still we will take the expression $f_{n} \approx f_{\text {on }}\left(1-\frac{\Delta m_{\text {fluid }}}{M_{\text {Prong }}}\right)$ as a cautious approximation, whose accuracy will have to be verified experimentally (as we do below); for simplicity we will consider only the fundamental resonance mode. But first, in anticipation to the experimentally observed non-linear variation of the resonance frequency $f$ with the immersion length $d$ (to be shown below), and to emphasize that the approximations employed above are valid for small values of $\Delta d$ (compared to the length of the TF), it is convenient to rewrite the expression above in the following form,

$$
\frac{\Delta m_{\text {fluid }}}{M_{\text {Prong }}}=\frac{\Delta f(d)}{f(d)}
$$

In this expression, at a given immersion length $d$ the resonance frequency is $f=f(d)$; an additional immersion length $\Delta d$ (controlled by the user) produces an additional fluid mass displacement $\Delta m_{\text {fluid }}$, which gives rise to a corresponding change in the resonance frequency $\Delta f$ $=\Delta f(d)$ detected in the SANM; all the quantities are subsequently updated for the next approximation. Given the rationale and approximations that led to obtain expression (1), the additional mass inertia model is then basically the description of a simple harmonic oscillator (SHO).

For a cylindrical probe of radius $r$ immersed in a fluid of density $\rho_{\text {fluid }}$, an increase in the immersion length by $\Delta d$, produces an additional displacement of fluid mass given by,

$$
\Delta m_{\text {fluid }}=\rho_{\text {fluid }} \Delta V_{\text {fluid }}=\rho_{\text {fluid }} \pi r^{2} \Delta d
$$

From (1) and (2), one obtains,

$$
M_{\text {Prong }}=\Delta m_{\text {fluid }} \frac{f}{\Delta f}=\rho_{\text {fluid }} \pi r^{2} f \frac{\Delta d}{\Delta f}
$$


Publishiag the quantities on the right side of (3) are under experimental control within the SANM system, which provides an opportunity to verify the validity of the SHO model being used. For measurement taken at different immersion length, we should expect to obtain a constant value for $M_{\text {Prong }}$. This is verified in the Analysis section below.

\section{RESULTS}

Table 3 shows a summary of order of magnitude changes in the probe's amplitude of oscillations (an indicator of damping effects), the probe's resonance frequency shift (an indicator of elastic effects) and acoustic signal (sound engender by the fluid and monitored by the SANM), which were obtained from systematic measurements performed with probes of different diameters and using droplets of different viscosities. The partial results quoted in the table correspond to behavior of the signals near the, arbitrarity selected, $160 \mu \mathrm{m}$ immersion length, just to obtain first a rough comparison among them. Notice that the values of the "resonance frequency shift" in column-3 and the "acoustic" signal in column-5 are somewhat close to each other, but both are quite different than the values in column-4 ("amplitude of oscillation"). This correlation (or lack of it) among these three signals turns out to be consistent across the full range of immersion length, 0 to $280 \mu \mathrm{m}$, as will be shown below. 
Publishitgle 3. Test Results

\begin{tabular}{|c|c|c|c|c|}
\hline Figure & Control & $\begin{array}{l}\text { Resonant } \\
\text { Frequency Shift }\end{array}$ & Oscillation amplitude & Acoustic \\
\hline Fig 2 & $\begin{array}{l}\text { Increasing } \\
\text { "immersion } \\
\text { length } d \text { " of the } \\
\text { probe in water } \\
\text { droplet. }\end{array}$ & $\begin{array}{l}\text { QTF resonance freq. } \\
\text { and acoustic peak } \\
\text { freq. shift together. }\end{array}$ & Resonance amplitude & Peak amplitude \\
\hline $\begin{array}{l}\text { Figs. } \\
3,4\end{array}$ & Probe diameter & $\begin{array}{l}\text { At } d=160 \mu \mathrm{m}: \\
45 \% \text { rate reduction } \\
\text { in frequency shift } \\
\text { per immersion } \\
\text { length due to a } 58 \% \\
\text { decrease of probe } \\
\text { cross section area. }\end{array}$ & $\begin{array}{c}\text { At } d=160 \mu \mathrm{m}: \\
\text { Amplitude decreases } \\
2 \% \text { more per } \\
\text { immersion length due } \\
\text { to a } 35 \% \text { decrease in } \\
\text { probe diameter. }\end{array}$ & $\begin{array}{c}\text { At } d=160 \mu \mathrm{m}: \\
67 \% \text { rate reduction in } \\
\text { acoustic signal per } \\
\text { immersion length due } \\
\text { to a } 58 \% \text { decrease of } \\
\text { probe's cross section } \\
\text { area. }\end{array}$ \\
\hline $\begin{array}{l}\text { Figs. } \\
5,6\end{array}$ & $\begin{array}{c}\text { Droplet } \\
\text { viscosity. }\end{array}$ & $\begin{array}{c}\text { At } d=160 \mu \mathrm{m}: \\
10 \% \text { rate increase of } \\
\text { frequency shift } \\
\text { caused by } \\
\text { a } 500 \% \text { increase in } \\
\text { viscosity. }\end{array}$ & $\begin{array}{c}\text { At } d=160 \mu \mathrm{m}: \\
\text { Amplitude decreases } \\
2 \% \text { less per immersion } \\
\text { length when the } \\
\text { viscosity increases by } \\
500 \% \text {. }\end{array}$ & $\begin{array}{c}\text { At } d=160 \mu \mathrm{m}: \\
20 \% \text { rate increase } \\
\text { in acoustic signal } \\
\text { due to } \\
\text { a } 500 \% \text { increase in } \\
\text { viscosity. }\end{array}$ \\
\hline Fig. 7 & Driving force & None. & None. & None \\
\hline Fig. 8 & $\begin{array}{c}\text { Water } \\
\text { evaporation } \\
\text { time } \\
\end{array}$ & & None. & None. \\
\hline Fig. 9 & Water volume & None & None. & None \\
\hline
\end{tabular}




\section{Publishing Probe immersed in a droplet of pure water}

Fig. 2 shows few representative spectral responses from the QTF and the acoustic sensor, both acquired simultaneously with the probe immersed in a drop of water.

(a)

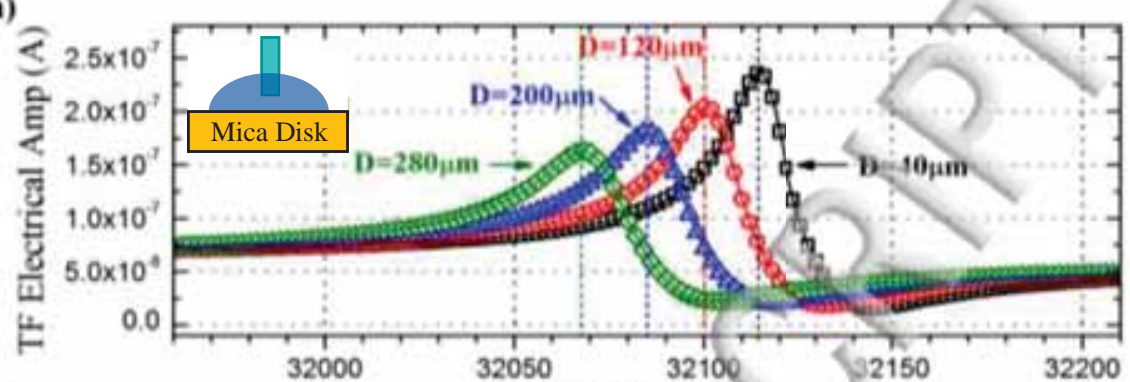

(b) Frequency $(\mathrm{HL})$

(c)
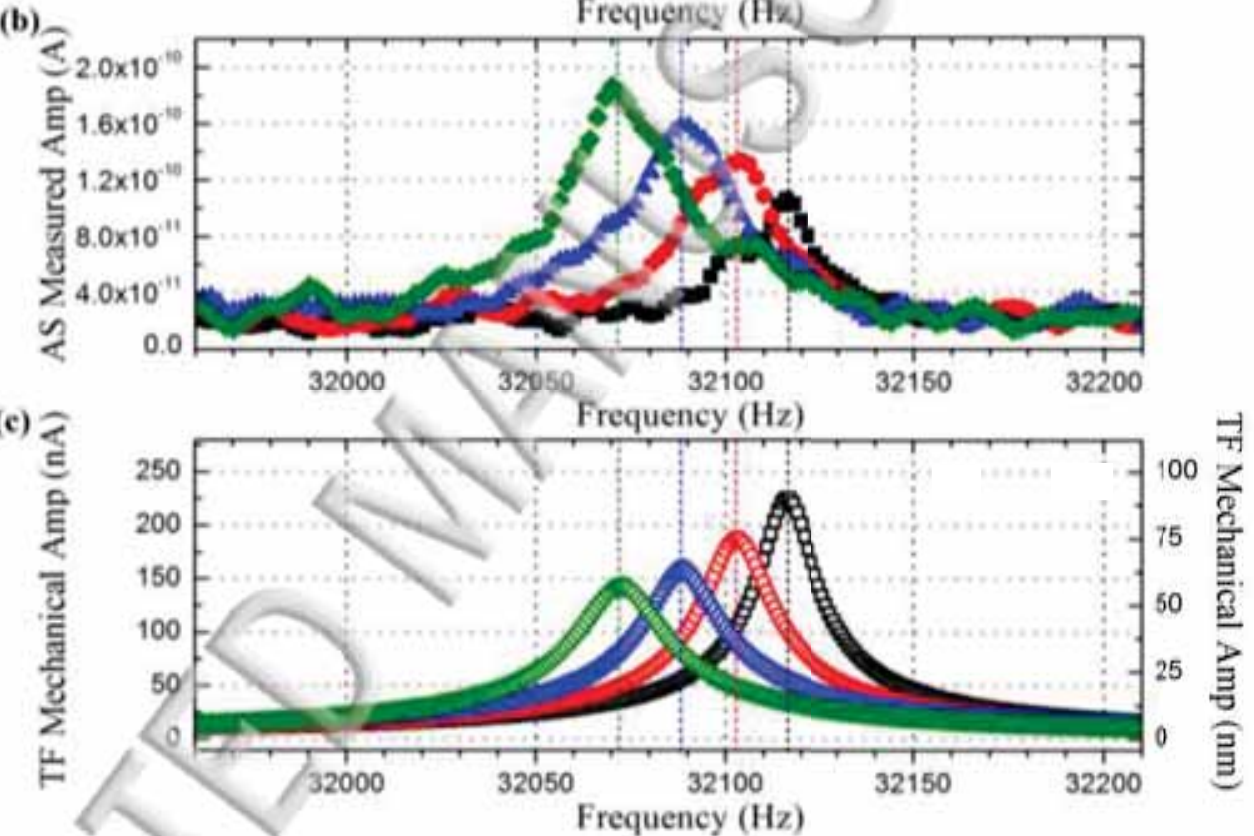

Fig. 2 Effects of probe immersion in a drop of water. a) Few representative electrical QTF spectra, and b) corresponding SANM acoustic response from the fluid, for a probe of $125 \mu \mathrm{m}$ diameter at 40, 120, 200, and $280 \mu \mathrm{m}$ immersion lengths respectively. Notice that both signals peak at the same frequency. c) Calculated mechanical oscillation-amplitude spectra obtained from a) after removing the effect of the QTF's intrinsic capacitance (as described in the text); the right vertical axis uses a $2.5 \mathrm{nA} / \mathrm{nm}$ calibration factor.

Notice that at each immersion length the peak frequency of both signals experience the same negative shift. (A similar feature is also observed in near-field measurements of mesoscopic fluid films using a SANM system, except that the frequency-shift is positive). ${ }^{5}$ The figure also shows 
Publishithegt at deeper immersion lengths the QTF peak amplitude decreases while the peak of the acoustic signal increases. For comparison, in the near field case a similar trend is observed at large probe-sample distances, but at smaller separation distances both decrease; ${ }^{6}$ the latter can be attributed then to effects caused by the substrate.

The results presented in Figures 3 and 4 confirm further that the signals vary monotonically and with smooth variations in the slope (i.e. non-linearly) over the entire 0 to $280 \mu \mathrm{m}$ immersion range. For comparison, in the near field case the variation in slope is not that predictable and, occasionally, abrupt changes are observed. ${ }^{20}$

\section{B. Effects of Probe Diameter}

Figures 3 and 4 show the changes in resonance frequency shift and resonance oscillation amplitude for probes of different diameter positioned at various submersion lengths inside a pure-water droplet. Each of the four traces corresponds to a new probe attached to a different QTF, which in general resulted in correspondingly different initial resonance frequency and resonance peak amplitude. For each approaching step, a CCD camera allowed observing the instant when the fiber probe gets in contact with the water boundary (as shown in Fig. 1b); this vertical position is defined as the $d=0^{+}$immersion length.

One parameter of interest is the negative resonance frequency shift (colloquially referred here also as "red shift") experienced by the probe as it gets progressively immersed into a $\sim 5 \mathrm{~L}$ water droplet. The shift is tracked relative to the resonance frequency measured when the probe is completely withdrawn from droplet (a location referred to as $d=0^{-}$). The slope of a given frequency-shit trace changes with immersion length. At $d \sim 160 \mu \mathrm{m}$, the rates of change are $20 \mathrm{~Hz}$ and $11 \mathrm{~Hz}$ per every $100 \mu \mathrm{m}$ immersion length for the $125 \mu \mathrm{m}$ diameter and $81 \mu \mathrm{m}$ diameter probes, respectively. This represents a $9 / 20=45 \%$ rate reduction in frequency shift per immersion length caused by a 58\% decrease in probe's cross section area. Figure 3 also shows that the rate at which the probe's frequency redshift changes per immersion length is the same as the rate at which the corresponding acoustic signal peak frequency changes. 

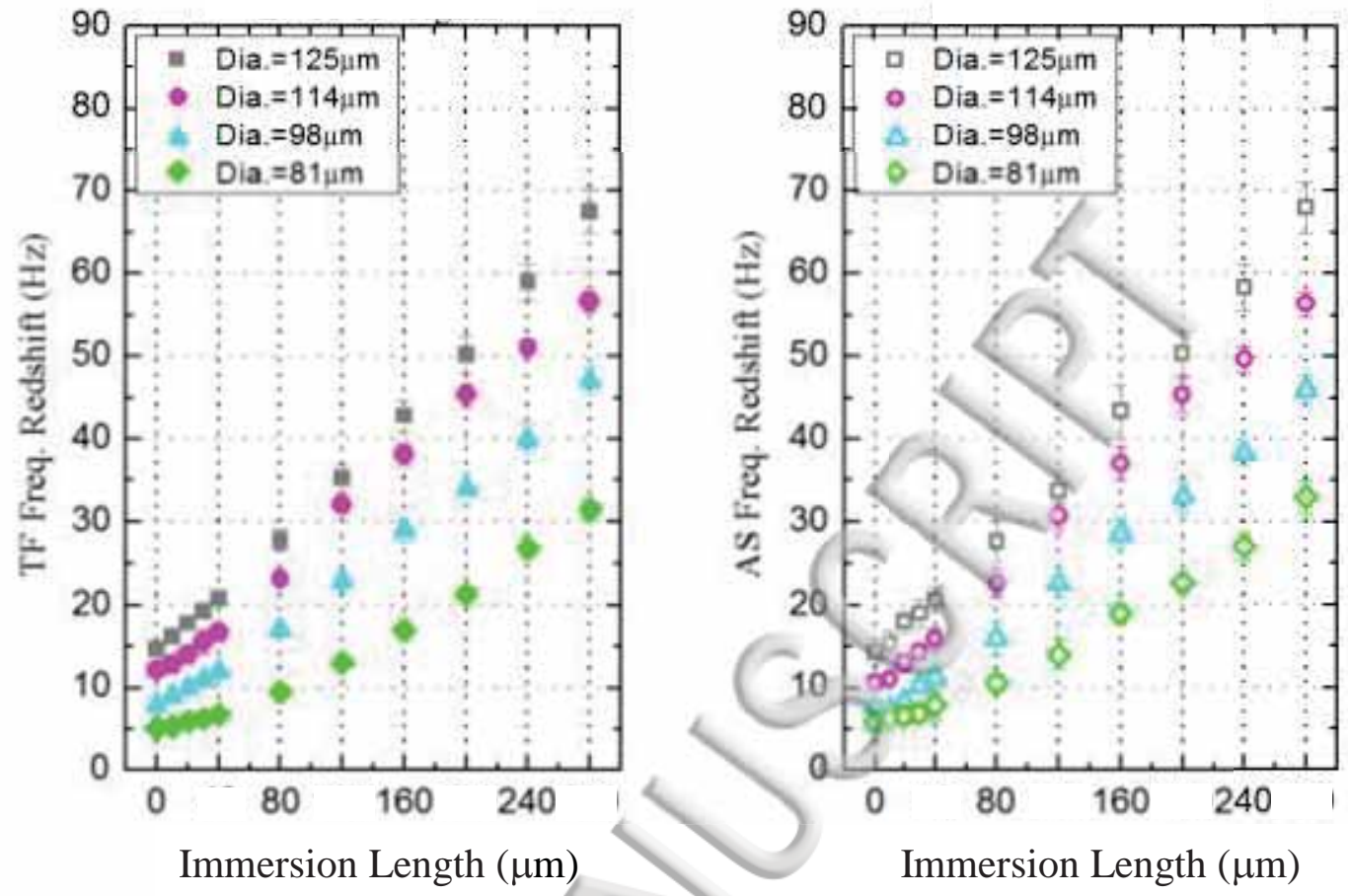

Fig. 3 Effects of probe diameter on the magnitude of the probe's resonant frequency shift (left) and on the corresponding magnitude of the peak frequency shift of the acoustic response (right), at various immersion length in a pure-water droplet.

In Figure 4, the diagram on the left shows the mechanical resonance amplitude at different immersion lengths for probes of different diameters. Notice the rate of resonance-amplitude reduction per immersion length is practically the same for each probe. Indeed, at $d \sim 160 \mu \mathrm{m}$ the thinnest probe decreases barely $2 \%$ more in amplitude than the thicker probe per $100 \mu \mathrm{m}$ immersion depth. In contrast, the rate at which the acoustic signal changes has a much stronger dependence on the probe diameter, as revealed by the diagram on the right side of Fig. 4. One observes a $67 \%$ rate increase in acoustic signal per immersion length when comparing the cases for the thinnest ( $81 \mu \mathrm{m}$ diameter) and the thickest (125 $\mu \mathrm{m}$ diameter) probe. There is then a markedly difference between the light damping effects on the probe (which, in a simple harmonic motion model, is revealed by the changes in the probe's oscillation amplitude) and the strong acoustic response from the fluid, both caused by the probe-fluid interaction.

In the reported acoustic traces, each value (output current from the acoustic transducer) has been normalized with the corresponding probe's oscillation amplitude (output current from the tuning fork sensor), hence giving values in "normalized acoustic units (A/A)". This 
Publishing nalization procedure allows comparing the strength of the acoustic signal obtained at different immersion lengths as if the probe were oscillating with the same amplitude in each single measurement. (The experimentally observed linear response of the acoustic sensor with the probe's oscillation amplitude, addressed in Sections IV.D below, justifies further this normalization procedure).
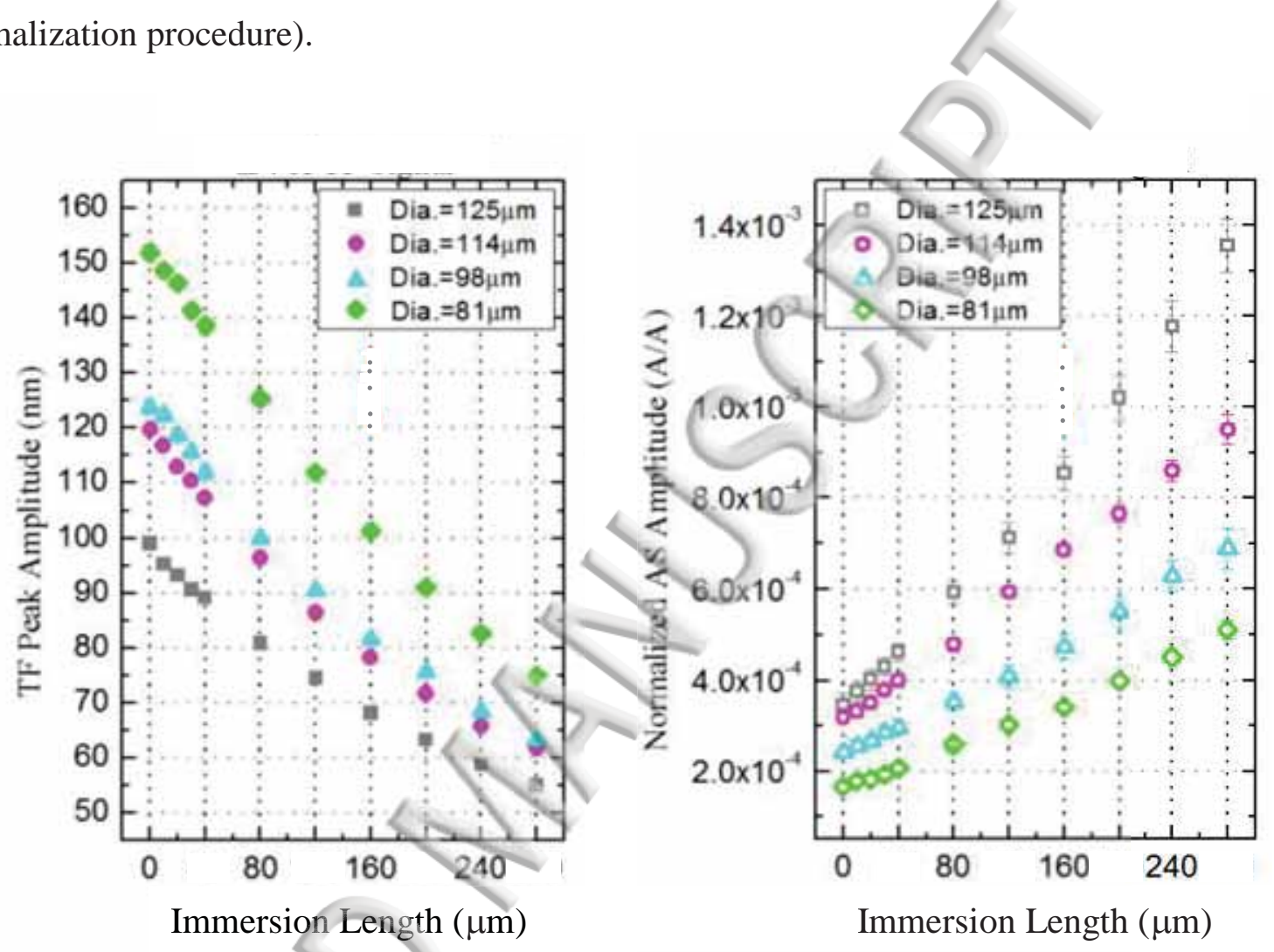

Fig. 4 Effects of probe's diameter on the probe's resonance amplitude (left) and on the acoustic signal response from pure water droplets (right) at different immersion lengths.

\section{Effects of fluid viscosity}

Figure 5 shows an increase in the the magnitude of the shift in the probe's resonance frequency (diagram on the left) and in the fluid's peak frequency acoustic response (diagram on the right) when the viscosity of the droplets increases. The results were obtained using a probe of $125 \mu \mathrm{m}$ diameter. At $d=160 \mu \mathrm{m}$ the changes in frequency are approximately $20 \mathrm{~Hz}$ and $25 \mathrm{~Hz}$ per every $100 \mu \mathrm{m}$ immersion length for the droplets of $0 \%$ and 50\% glycerin concentration respectively. In the latter case we have to factor out the frequency increase due to the larger density of the more viscous fluid, which results in a net $22 \mathrm{~Hz}$ increase instead (just due to 
Publishinigcosity $)^{21}$. The $2 \mathrm{~Hz}$ difference reflects a 10\% difference in frequency-shift due to a change from $0 \%$ and $500 \%$ glycerin concentration. Changes in the peak frequency for the acoustic signal are shown in the right side diagram of Fig. 5; notice it is practically a replica of the diagram on the left.
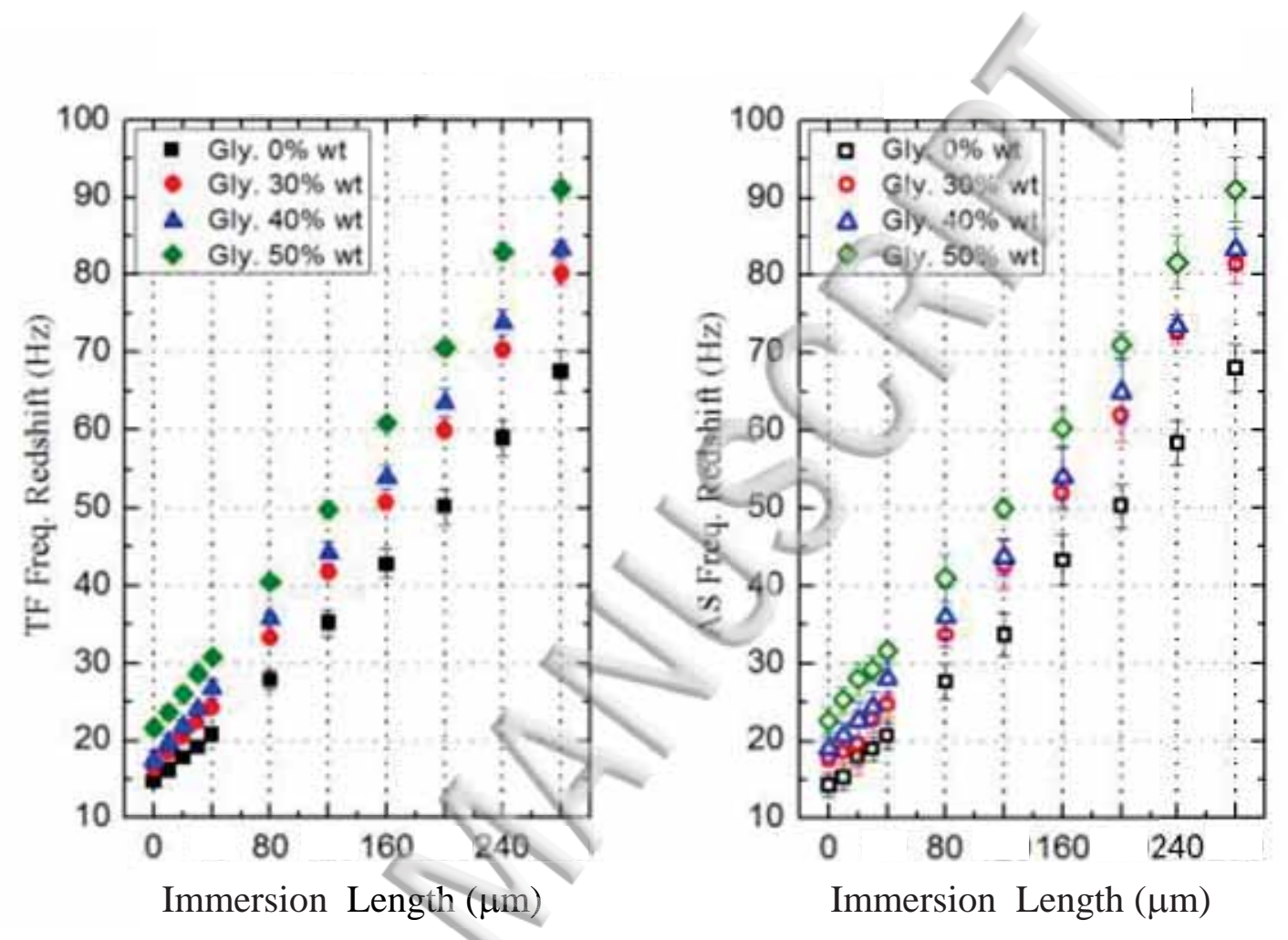

Fig. 5 Effects of droplet viscosity on the magnitude of the probe's resonant frequency shift (left) and on the corresponding frequency at which the acoustic signal registers a peak in its amplitude, as a function of the submersion length. The diameter of the probe is $125 \mu \mathrm{m}$ in all the cases.

In Fig. 6, the diagram on the left shows the changes in the probe's resonance amplitude for droplets of different glycerin concentrations. Notice, the rate of amplitude reduction per immersion length is practically independent of the viscosity; near $d=160 \mu \mathrm{m}$ immersion length, there is a $2 \%$ difference when comparing the traces corresponding to $0 \%$ and $500 \%$ glycerin concentration. In contrast, the diagram on the right side of Fig. 6 shows a much stronger dependence of the acoustic signal strength on viscosity. Near $d=160 \mu \mathrm{m}$ immersion length, there is a $20 \%$ change in acoustic signal due to a $500 \%$ increase in viscosity, indicating that there is an effective contribution from the viscous nature of the fluid to the production of sound. Again, the 
Publishiqugsed acoustic signal values are given in "normalized acoustic units (A/A)" as described in the previous section.
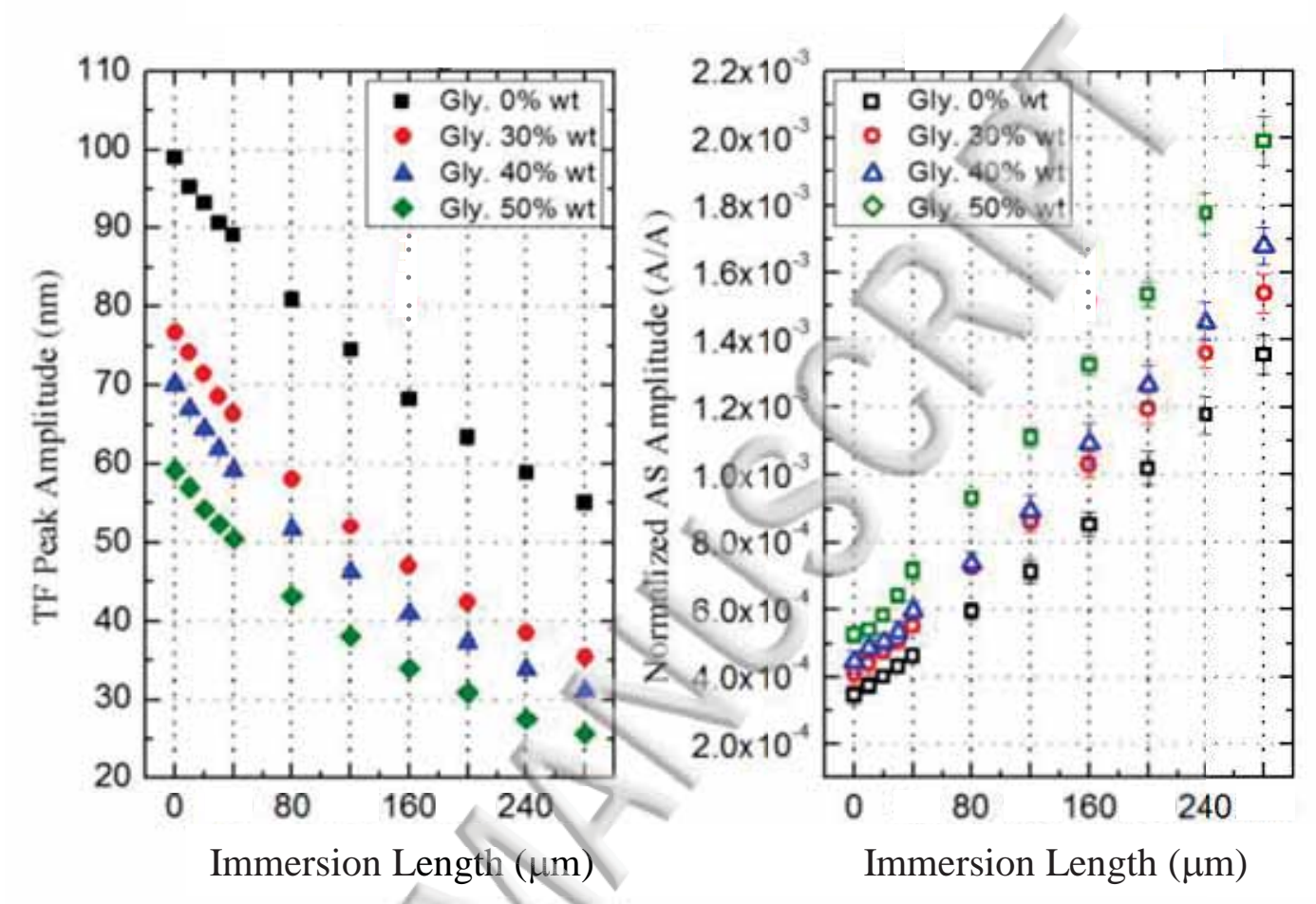

Fig. 6 Viscosity effect on the probe's resonance amplitude (left) and on the corresponding acoustic response from the fluid (right) as a function of the immersion length using the same probe diameter in liquids of different viscosities.

\section{Effect of the driving voltage}

Figure shows the response from the QTF (top graph) and the acoustic sensor (center graph) as a function of the driving voltage set by the signal generator (see also Fig. 1). The observed linear response adds reliability to normalization processes of the acoustic signal (bottom graph), where the output current from the acoustic transducer has been divided by the corresponding probe's oscillation amplitude; this results in "normalized acoustic units (A/A)". 


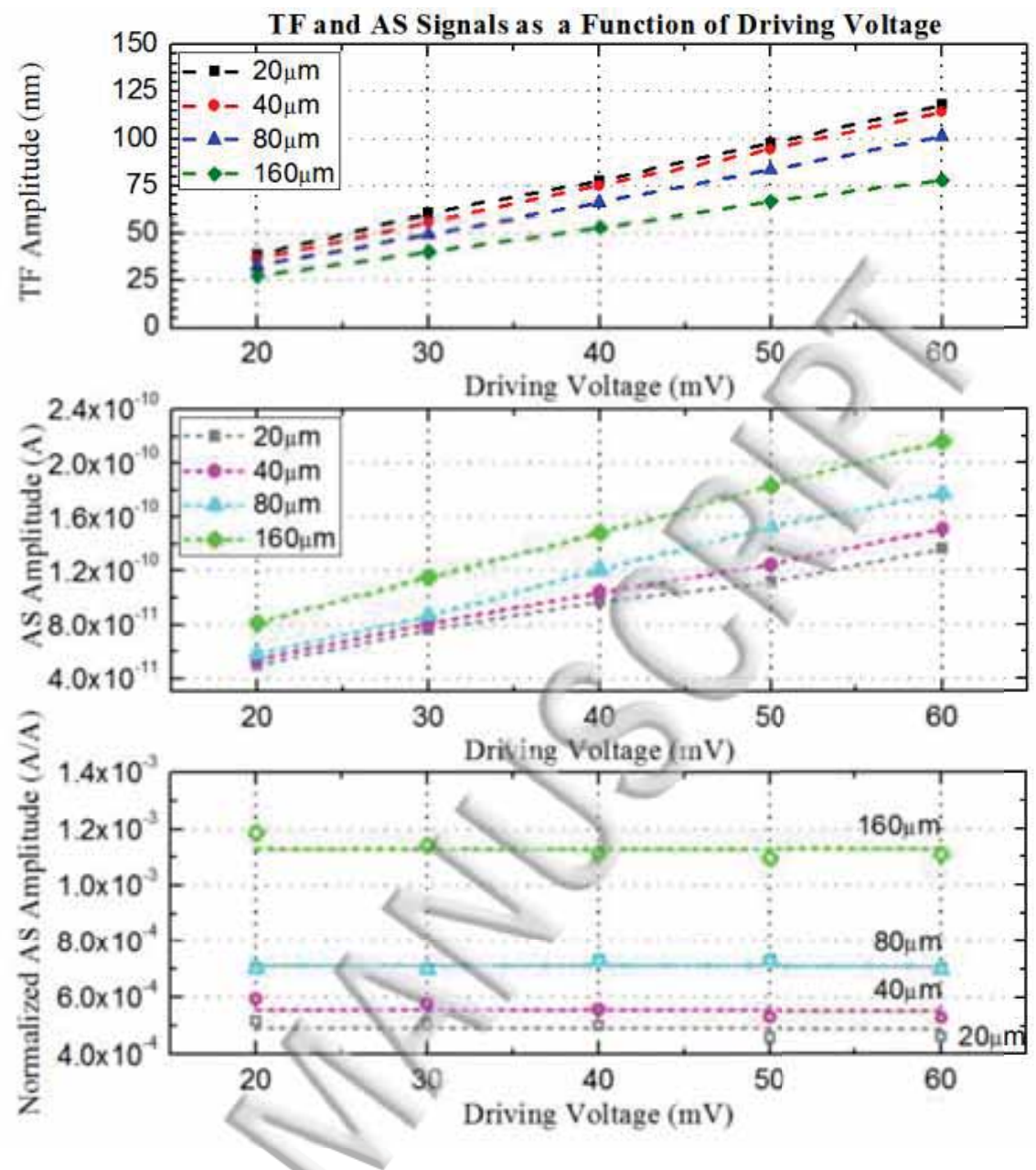

Fig. 7 Linear response from the QTF (top) and acoustic (center) sensors to increasing values of the excitation source driving voltage amplitude.

\section{E. The effect of liquid evaporation during experiments}

The results in Fig. 6 evaluate whether or not the liquid evaporation was a detrimental factor during the measurements. As the drop of liquid evaporates, the amount of liquid in contact with the probe would decrease and thus cause an increase in the probe's resonance frequency, which would convolute the reported results. To evaluate this effect, we recorded the vibration spectra of the QTF and acoustic signals with a probe kept at fixed position (80 $\mu \mathrm{m}$ immersion length into the initial water droplet). In Figure 6, the time interval between consecutive traces is 1 minute. The entire recording lasted $\sim 5$ minutes, which is much longer than the average time employed to run a given subset of the experiments described in the sections above. No significant change in the resonance frequency shift due to the evaporation during this interval of time is observed. 


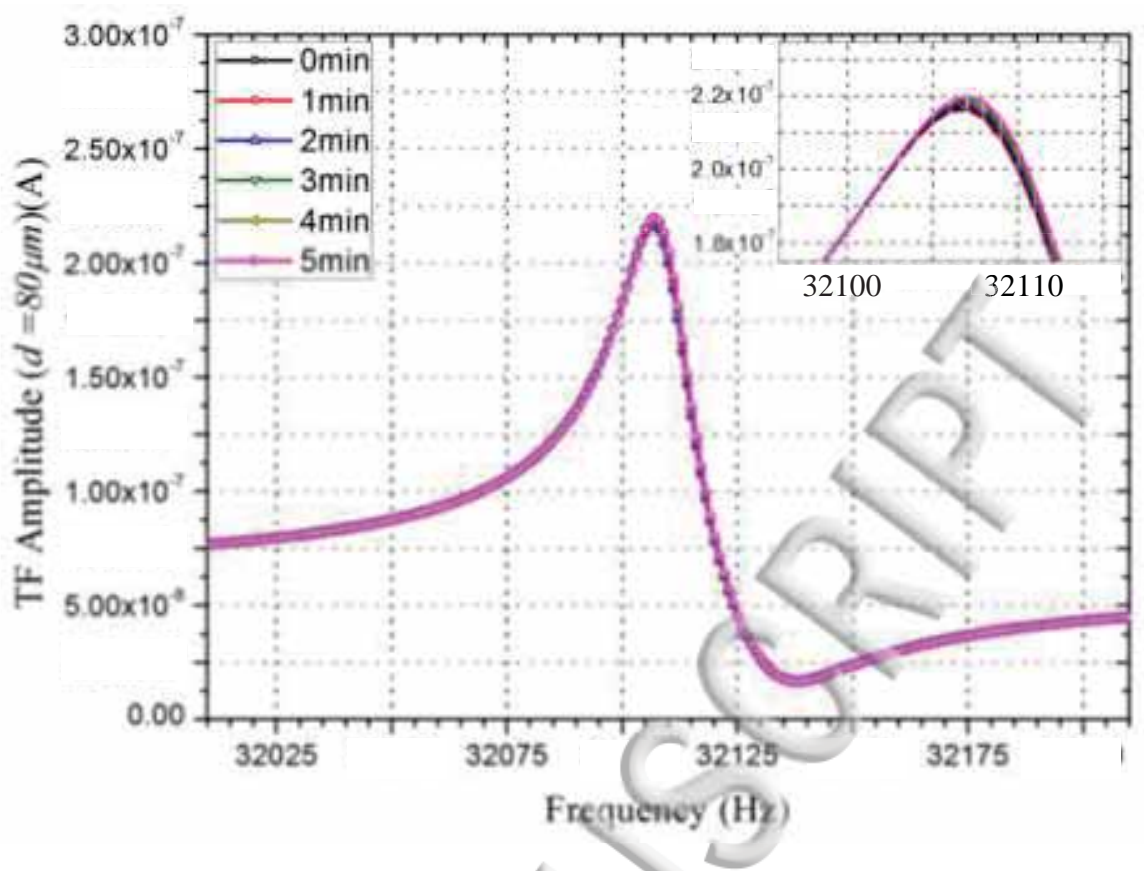

Fig. 8 Multiple recordings of the QTF frequency response while keeping the laterally oscillating probe at a fixed immersion length in distilled water. The time interval between two traces next to each other is $1 \mathrm{~min}$, and the entire process lasted $\sim 5 \mathrm{~min}$. A more detailed position of the resonance peaks is shown in the inset.

\section{F. Effects of the Liquid Droplet Volume}

The baseline test condition is to use a droplet of consistent $5 \mu \mathrm{L}$ volume. But we wanted to explore whether the exact volume could have an effect on the peak frequency shift of the acoustic response, and hence, affect the reproducibility of the results reported above. Also, using droplets of different volumes places the air-fluid interface at different distances from the substrate, which allows evaluating a potential influence, if any, of the substrate on the reported results. Figure 9 shows the reproducibility of the QTF and acoustic responses when using different droplet volumes ( $5 \mu \mathrm{L}, 7.5 \mu \mathrm{L}, 10 \mu \mathrm{L}$. $12.5 \mu \mathrm{L}$ and $15 \mu \mathrm{L}$ ). In each case a $125 \mu \mathrm{m}$ diameter probe was submerged $80 \mu \mathrm{m}$ into the droplets. No effect of the droplet volume on the resonance frequency is observed. 


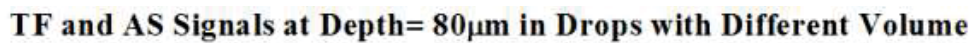

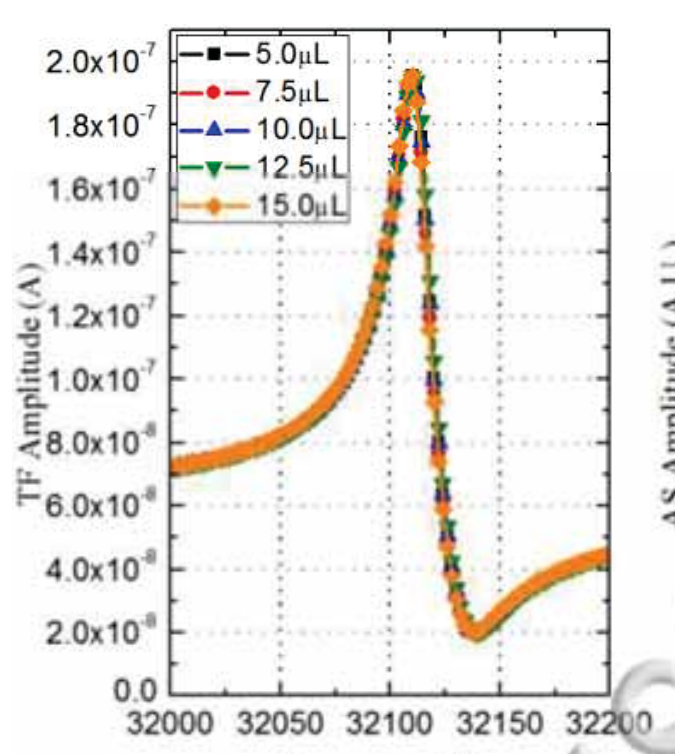

Frequency $(\mathrm{Hz})$

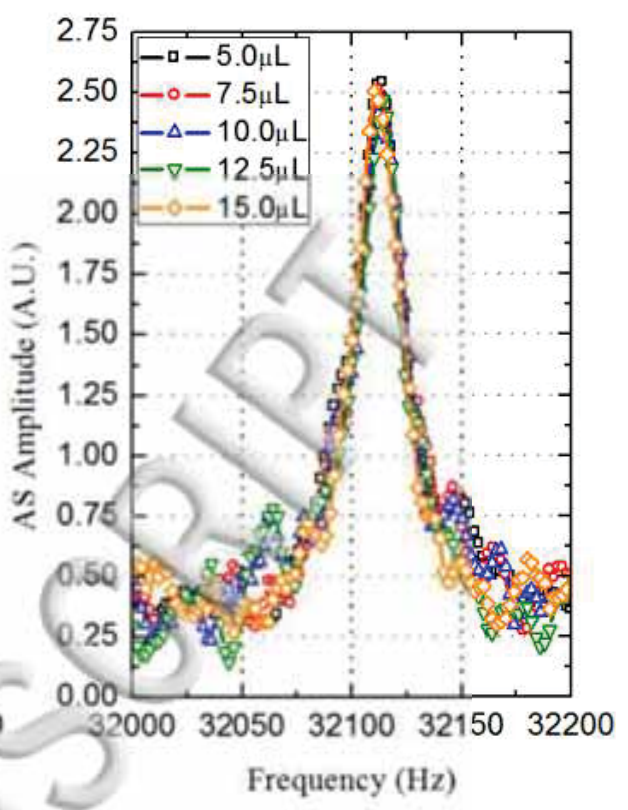

Fig. 9 QTF electrical response and fluid's acoustic response from droplets of different volumes.

\section{ANALYSIS}

\section{A. Signatures of simple harmonic oscillatory motion in the probe's response}

Figure 10 shows responses from the QTF sensor, which reveal the probe behaves as a simple harmonic oscillator (SHO). Figure 10a shows that the rate at which the mechanical quality factor Q changes with immersion length is practically the same whether the probe is in a droplet of pure water (1.005 centipoise viscosity) or in a droplet of 50\% glycerin concentration (6.00 centipoise viscosity). The results suggest that viscosity does not play a significant role as energy dissipation channel in the probe-fluid interaction. A plausible explanation considers the liquid molecules adhering to the surface of the laterally oscillating probe upon its entrance into the droplet (a manifestation of the zero-slip hydrodynamic condition effect, which happens to be valid also on hydrophobic substrates ${ }^{22}$ ). In consequence, the amount of liquid set into motion (and eventually constituting a wave traveling in a direction transverse to the lateral oscillations) resides mainly inside a boundary layer surrounding the probe. That layer has a viscosity-dependent thickness of just a few micrometers (as estimated in Section V.E below). The small value of the boundary layer's thickness (compared to the probe diameter) and the lack of relative sliding motion at the 
Publishisofid-liquid interface (which otherwise would affect the probe's amplitude more strongly) diminishes the damping effects of viscosity.

This interpretation also helps to put in context the role of microscopic friction in the implementation of the zero-slip condition. As currently accepted, the energy dissipation raised by the viscous resistance is at the mesoscale (of the order of the boundary layer's thickness), while that raised by the molecular friction, i.e. liquid molecules adsorb/desorb on solid atoms, is at the microscale. $^{23,24}$ First, the independence of amplitude damping per immersion length on viscosity indicates that the zero-slip condition is strictly in place; otherwise (as argued above) a higher viscosity would cause a higher damping rate. Second, the observed larger change in amplitude when the probe just gets immersed into a fluid of higher viscosity (as indicated by the arrows along the horizontal axis in Fig. 10b) illustrates further the effects of microscopic friction. Notice, more energy is dissipated on the 50\% glycerin fluid (amplitude deceases down to 30\%) compared to the immersion in pure water (amplitude decreases down to 50\%).

The weak effect of viscosity on damping once the probe is immersed into the few micro-liter volume fluid (as reported here) contrasts with its, currently controversial, role on the dynamics of mesoscopic fluids. On one hand, some reports suggest that viscosity increases as the fluid gets progressively more confined. ${ }^{25}$ Two related mechanisms may contribute to this increase in viscosity. First, the small probe-substrate gap $(<15 \mu \mathrm{m})$ compared to the $125 \mu \mathrm{m}$ diameter of the probe (as in the case of Ref. 25) causes constrains in the motion of the confined fluid. Also, since such a small gap fall in the thickness range of the fluid boundary layer, the probe and substrate boundaries may have a significant effect of the dynamics of the trapped fluid. Second, adhesion forces attract the fluid molecules towards the probe and substrate solid boundaries (imposing the zero-slip hydrodynamic condition), which results in larger velocity gradients inside the gap. Both mechanisms may contribute to have a mesoscopic fluid with an "effective viscosity" much larger than the viscosity of bulk fluid. On the other hand, there also exist reports claiming that confinement does not affect the viscosity of mesoscopic fluids at all. ${ }^{26}$ A resolution of this controversy has been presented more recently based on experiments that test the behavior of mesoscopic fluids (confined between a mica surface and a silicon-oxide probe) at different confinement speeds. ${ }^{27}$ No variation in viscosity is observed due to both confinement and molecular ordering near an atomically flat surface when the mesoscopic fluid is probed at high 
Publishimged (1.5 nm/s confinement rate). At this high speeds, when molecules are in an ordered state cannot easily move out of the gap as a group, thus becoming "stuck" and responding elastically to external shears; i.e. the mesoscopic fluid behaves solid-like. ${ }^{27}$ Higher viscosities are however measured when the fluid is probed at lower speed $(<0.6 \mathrm{~nm} / \mathrm{s})$ and due to the restricted motion imposed by confinement (as described at the beginning of this paragraph); the liquid behaves liquid-like, but with enhanced viscosity ${ }^{27}$

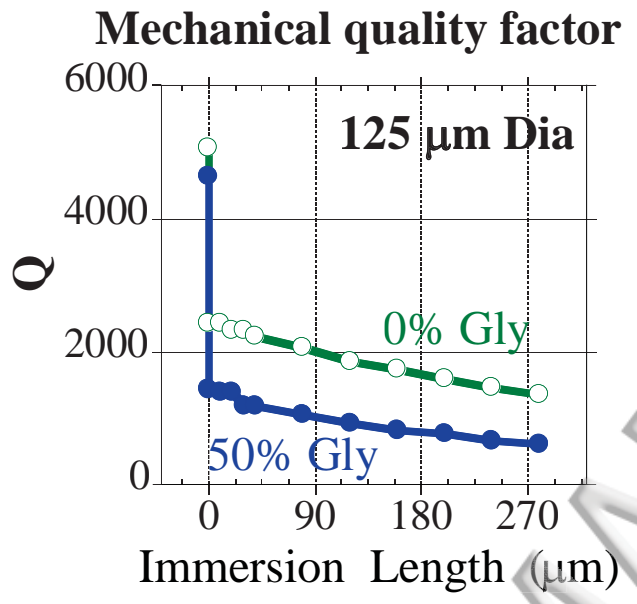

a)

\section{Q vs Amplitude}

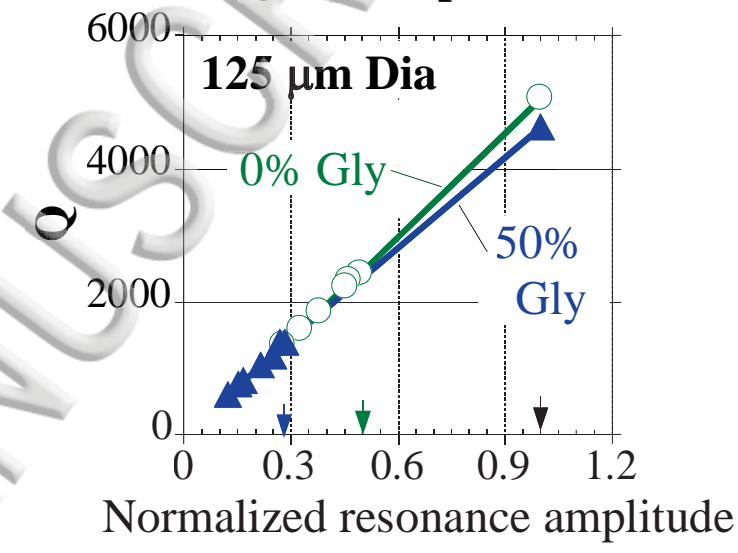

b)

Fig. 10 Variation of the mechanical quality factor Q with a) probe's immersion length, and b) probe's resonance amplitude (at different immersion length). Two cases are presented, pure water (open circles trace) and 50\% glycerin (solid circles trace) droplets; in both cases the probe diameter is $125 \mu \mathrm{m}$. The arrows along the horizontal axis in 10b) indicated the normalized probe's amplitude right after the tip immerses into the corresponding fluid. The behavior in both graphs display signatures of a simple harmonic oscillator motion (as described in the text).

Fig. 10b displays the mechanical $Q$ factor as a function of the probe's amplitude (measured with the probe first completely outside the droplet and then at a series of different immersion positions) corresponding to two different droplets of $0 \%$ and $50 \%$ glycerin concentration, respectively. Notice the linearity between $Q(\sim 1 /($ damping constant $))$ and the probe's resonance amplitude is maintained whether the probe is immersed or not in the droplet. That is, as far as the probe is maintained at resonance, we observe that the net damping force $\left(F_{\mathrm{D}} \sim\right.$ damping constant $\times$ amplitude $\sim$ amplitude/Q) remains constant at different immersion lengths. It is as if the 
Publishiarg litude and corresponding damping constant confabulate to keep the total damping force the same while the probe gets immersed into the droplet. ${ }^{14}$ The latter is a signature of a simple harmonic oscillator motion (SHO). This further justifies the use of the SHO, adopted below, to describe the additional experimental results.

\section{B. Validation of the additional inertial mass model}

Fig. 11 shows calculated values for $M_{\text {Prong }}$ predicted by expression (3) above, $M_{\text {Prong }}=\rho_{\text {fluid }} \pi r^{2} f \frac{\Delta d}{\Delta f}$, where we have used values for $\Delta d$ and $\Delta f$ measured with the probe placed at different immersion lengths in a droplet of pure water. Calculations were performed for probes of three different diameters. Notice that for the probes of $125 \mu \mathrm{m}$ diameter ("rhombus" trace) and $98 \mu \mathrm{m}$ diameter ("triangles" trace) the calculated values consistently lie around $M_{\text {Prong }}=(2 \pm 0.5) \times 10^{-6} \mathrm{Kg}$. The case for the $81 \mu \mathrm{m}$ diameter ("open circles" trace) shows more discrepancy, but still with a tendency to fit around a constant value. (The discrepancy may be due to the fact that, being the thinnest probe protruding $\sim 1 \mathrm{~mm}$ beyond the prong, that fiber section may undergo additional bending, a situation that departs from the the assumed oscillation of a fully stiff prong.)

The calculated value of $M_{\text {Prong }}$ turns out to be very sensitive to the precise measurement of the immersion length. This sensitivity is exposed by the three "rhombus" traces in Fig 11; they were obtained by/purposely/introducing $1 \mu m$ uncertainty in the immersion length $d$, which leads to an uncertainty of $0.2 \mu \mathrm{Kg}$ (see error bar segment at the bottom-left side of the figure as a reference). In spite of this sensitivity, it is remarkable that all the calculated values accumulate around the $2 \mu \mathrm{Kg}$ mark for each submersion length and for three different probes. An alternative procedure to estimate the value of $M_{\text {Prong }}$ is to calculate the slope of the curve $\Delta f$ vs $\Delta d$ in Fig. 11. For the case of pure water, at $d=160 \mu \mathrm{m}$ the slope is equal to $\Delta d / \Delta f=200 \mu \mathrm{m} / 40 \mathrm{~Hz}$, for which expression (3) gives $M_{\text {Prong }}=\rho_{\text {fluid }} \pi r^{2} f \frac{200 \mu m}{40 ~ H z}=2 \times 10^{-6} \mathrm{Kg}$. For comparison, the mass of one prong of the QTFs used here [of dimensions $L=(3.8 \pm 0.01) \mathrm{mm}, W=(0.35 \pm 0.01) \mathrm{mm}, T=$ $(0.6 \pm 0.01) \mathrm{mm}$; quartz density $\rho_{\text {quartz }}=2650 \mathrm{~kg} / \mathrm{m}^{3}$ ] has a mass equal to $M_{\text {Prong }}=(2.1 \pm 0.1)$ 
Publishipggg. Thus the value obtained from the hydrodynamic measurement matches very well the actual mass of one tine. Beyond this surprising accuracy predicting the value for $M_{\text {Prong }}$ (given the approximations made though our calculations), what we highlight here is the consistent constant value obtained for $M_{\text {Prong }}$ (at different probe's submersion lengths and for three different probes), which validates the use of a harmonic oscillator model, expression (1).

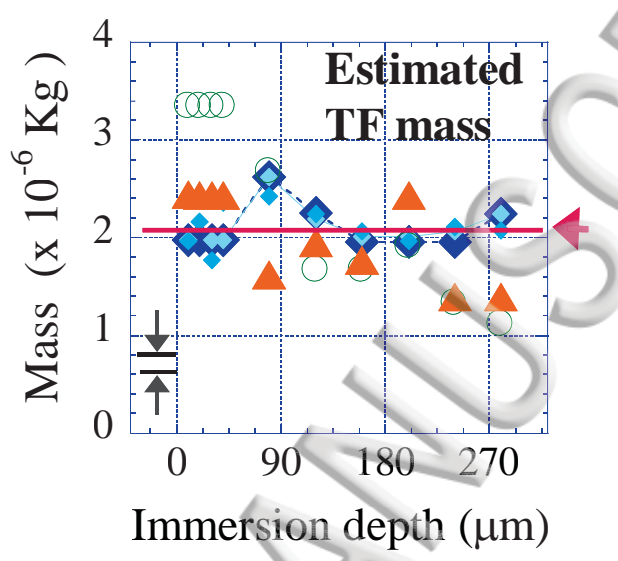

Fig 11 Calculated value of the QTF mass $M_{T F}$ predicted by expression (1) using the experimental values of the frequency shifts measured at different immersion distances. Data include results for three probes of different diameters, $125 \mu \mathrm{m}$ (rhomboids), $98 \mu \mathrm{m}$ (triangles), $81 \mu \mathrm{m}$ (circles). The symbol on the lower left side is to indicate that a $1 \mu$ m uncertainty in the immersion length $d$, produces an uncertainty of $0.2 \mu \mathrm{Kg}$ in the calculated mass. The horizontal line drawn at 2.1 $\mu \mathrm{Kg}$ is to indicate the actual mass of the TF prong.

\section{Frequency shift values expected from the observed changes in the mechanical factor $\mathbf{Q}$}

In addition to the mass loading effect, a change in the probe's resonance frequency can occur also as a consequence of damping effects. From a simple harmonic oscillator model, the amplitude peaks at $f=f_{o}\left(1-\frac{1}{2 Q^{2}}\right)^{1 / 2}$, which for $f_{o}=32,000 \mathrm{kHz}$ and $Q=2500$ gives $\Delta f \approx \frac{\Delta Q}{10^{6}} \mathrm{~Hz}$. Figure 10a shows a change from $Q_{2}=2,500$ to $Q_{1}=1,500$ when the probe is 
Publishisig nerged from $d=0^{+}$to $d=280 \mu \mathrm{m}$. For the observed change of $\Delta Q=1,000$, the expression above predicts a frequency shift $\Delta f$ in the order of $10^{-3} \mathrm{~Hz}$, much smaller than the observed $10 \mathrm{~s}$ of $\mathrm{Hz}$ reported in Fig. 3. This indicates that the damping effect is not the main source for the observed changes in the resonance frequency. Similar changes in the value of $Q$ are observed in near-field experiments (measured before and after the tip starts to interact with the sample's adsorbed layer) but the frequency shift is positive and of the order of $10 \mathrm{~Hz} ;{ }^{28}$ this suggests that damping is not the origin of the frequency shift. In short, for the bulk and mesoscopic fluid cases (both treated within the SHM model), a large change in $Q$ does not contribute significantly to the observed change in the probe's resonance frequency.

\section{Effects of probe diameter and droplet viscosity}

\section{Impact of probe diameter}

Fig. 3 shows that the rate at which the frequency-shift changes per immersion length is greater for thicker probes. The interpretation is straightforward; for larger cross section areas, a larger amount of water volume and mass will be driven and, according to the inertial mass model, a larger decrease in frequency shift will be observed. Driving a larger volume of water would also cause a stronger acoustic signal, which is verified in Fig. 3. On the other hand, Fig. 4 shows the rate of change in oscillation amplitude (which is associated to the damping effects) is much less pronounced, with a tendency to be practically the same for all the probes tested. A rate of amplitude change almost independent of the probe diameter can be explained by the fact that the probe is shaken laterally; hence the damping effects are caused mainly by the probe's lateral walls. As the probes get deeper immersed, all the probes increase their submerged lateral wall size in the same amount, hence contributing to the damping independent of their thickness.

In short, an increase in the probe diameter produces a larger rate of frequency shifts per immersion length, a larger rate in increasing acoustic signal, and an almost invariant resonance amplitude.

\section{Effects of fluid viscosity}

Figures 9 and 10 show that increasing values of fluid viscosities produce $i$ ) very small changes in the rate at which the oscillation amplitude decreases with immersion length, ii) large 
This manschipt was accepted by Phys. Huids. Click hine to see he version of record.

Publishingfiation in the rate at which the frequency-shift decreases with immersion length, and iii) a stronger acoustic signal. These results provide further indication that the damping forces inside the droplets play a weak role in the probe's motion. The minor change in amplitude despite the $500 \%$ change in viscosity can again be explained by considering an absence of relative sliding at the solid-water interface (the zero-slip condition). The dissipation occurs instead within the boundary layer that extends just a few microns from the solid probe buundary (for all the viscosities considered here). This region is small enough that an increase of viscosity by 5 times does not change the rate at which the oscillation amplitude yaries. In contrast, the larger change in frequency-shift indicates that a greater amount of fluid is dragged by the probe when immersed in droplets of higher viscosities. Such a feature is very revealing. It invites to consider that a similar mechanism could also be present in the case of near-field (probe-fluid-substrate) interactions, except that in the latter case one has to take into account that the fluid is not free to move (like in the bulk state) but restricted in its motion by their stronger attraction to the probe and substrate boundaries (fulfilling the zero-slip boundary condition). When the two solid boundaries become very close to each other (nanometer separation distances) a restoring force on the probe could then take place and, hence, cause a blue-shift in the probe's resonance frequency. Further, the reaction force on the (viscous) fluid would engender an acoustic signal. Such a correlation between the probe's frequency shift and the acoustic signal from the fluid in nearfield experiments has been addressed before. ${ }^{5}$ The more systematic tests, reported here, using fluids of different viscosities (although with a more sizable volume of fluid) support such earlier findings. We underscore then the effective contribution to the production of sound from the viscous nature of the fluid (the higher the viscosity, the greater the volume of the dragging fluid, and the greater the acoustic signal).

In summary when the probes get immersed into a fluid droplet the acoustic signal is consistently stronger when using either probes of larger diameter or fluids of greater viscosity; the probe's resonance frequency shift follows a similar trend. The resonance amplitude of oscillation, however, is weekly dependent on the probe diameter and fluid viscosity.

\section{E. The boundary layer effect}

As argued above, an additional contribution to the negative frequency shift comes from the motion of water molecules contained in the boundary layer neighbor to the probe's walls. The 
Publishing gocity field induced by a probe oscillating at frequency $\omega / 2 \pi$ establishes a wave that propagates in the direction perpendicular to the oscillations. They are, however, rapidly damped. The dampening is exponential, with a depth of penetration being given by, ${ }^{15,29}$

$$
\delta=\left(\frac{2 \gamma / \rho}{\omega}\right)^{1 / 2}
$$

where $\gamma$ is the dynamic viscosity and $\rho$ is the density of the fluid. For the $32 \mathrm{kHz}$ operating frequency in these experiments, $\delta$ varies from $3 \mu \mathrm{m}$ to $7.5 \mu \mathrm{m}$ for the $0 \%$ and $50 \%$ glycerin concentration respectively. The motion of this extra layer of water makes the probe a bit "thicker". The displaced mass increases by a factor $2 \pi r \delta / \pi r^{2}=2 \delta / r$, which causes an increase in the change of the resonance frequency according to the additional inertial mass model. In Section IV.C above we report $20 \mathrm{~Hz}$ and $25 \mathrm{~Hz}$ increases in frequency shift, per 100 $\mu \mathrm{m}$ immersion length, for the droplets of $0 \%$ and $50 \%$ glycerin concentration respectively, when using a probe of $125 \mu \mathrm{m}$ diameter. For the $0 \%$ concentration case, the factor $2 \delta / r$ is equal to 2 $\times 3 / 62.5=0.1$, which gives a $20 \mathrm{~Hz} \times 0.1=2 \mathrm{~Hz}$ contribution to the frequency shift per $100 \mu \mathrm{m}$ immersion length. For the $50 \%$ glycerin concentration droplet that factor is $2 \times 7.5 / 62.5=0.24$, which gives $25 \times 0.24=6 \mathrm{~Hz}$. The estimated $4 \mathrm{~Hz}$ difference matches well the $5 \mathrm{~Hz}$ experimental results reported in Section IV.C.

In short, the contribution (in the order of $\mathrm{Hz}$ ) to the change in the probe's resonance frequency from a boundary layer (whose thickness depends on the viscosity) is definitely much higher than the contribution expected from the damping effects (in the order of $\mathrm{mHz}$, as estimated in Section V.C above).

\section{F. Correlation between the probe's frequency shift and the fluid's acoustic response}

The results above indicate that for probes of increasing diameter and fluids of increasing viscosity $i)$ the rate of changes in resonance frequency and the acoustic signal with immersion length consistently become larger, but $i$ ) the rate of change in resonance amplitude (ascribed to dissipative effects) remain approximately constant. These findings invite to further examine how close a given pair of any of these signals correlates with each other. Such a comparison is presented in Figure 12, which displays our attempts to $a$ ) linearly fit the decreasing amplitude of 
Publishiosf signal to the increasing magnitude of the frequency-shift, as a function of the immersion length. To implement this comparison, the signals were simply multiplied by a corresponding constant factor (optimized for the best fitting) and then shifted so that the four traces could be displayed in a single graph. The fitting process was performed for probes of three different diameters.

According to the SHO model, signatures of increasing dissipative effects should be revealed by a decrease in the probe's resonance amplitude, as well as by a linear decrease of the mechanical factor $Q$ with decreasing resonance amplitudes; the latter is indeed observed in Fig. 10b above. Here we also observe that, as the probe immerses deeper into the droplet, both the resonance frequency and the resonance amplitude decrease (Figs. 3 and 4). But the results displayed in Fig. 12 show that the frequency and amplitude variations are not related linearly (the concavity of their corresponding traces are actually opposite). In contrast, the changes in acoustic signal fit remarkably close to the changes in frequency shift for each of the three different probes. Incidentally, such a correlation between the probe's resonance frequency shift and the fluid's acoustic response has been previously reported in near-field experiments performed with the SANM system. ${ }^{5}$ 
Probe Dia. $125 \mu \mathrm{m}$

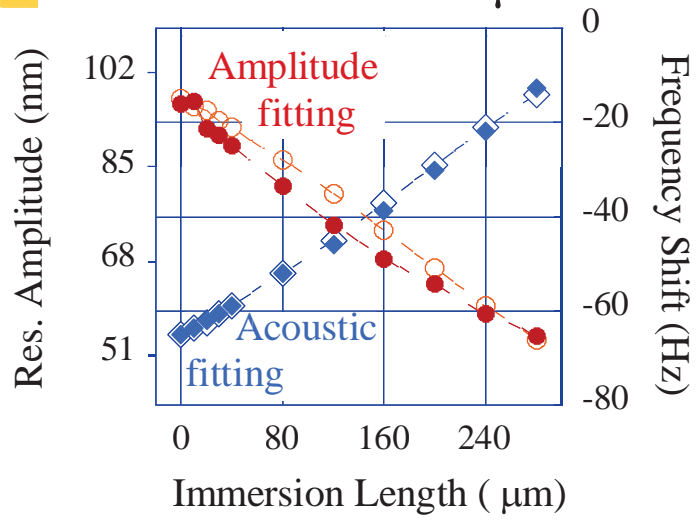

a)

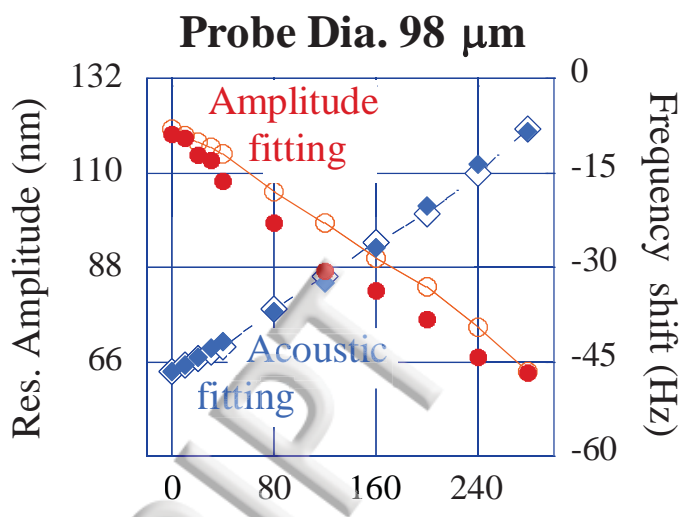

Immersion Length ( $\mu \mathrm{m})$

b)

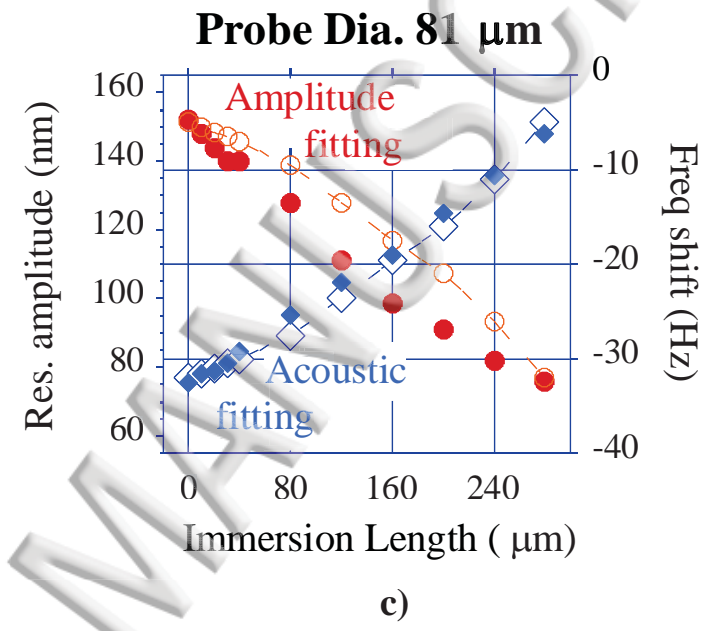

Fig. 12 Traces of the probe's resonance frequency shifts (open rhombus and open circles) fit well to a linear fitting process with the fluid's acoustic response (solid rhombus trace) but not to the changes in the probe's resonance amplitude (solid circles).

The result described in the previous paragraph, together with the experimental confirmation of expression (1) that relates the change in displaced fluid mass to the frequency shift (Fig. 11), offers a clear picture about the generation of acoustic signal: conversion of mechanical energy from the oscillating probe into fluid motion (sound) inside the droplet (which then couples into the sample substrate and reaches the acoustic sensor, as shown in Fig. 1 above). Fig. 12 indicates that this mechanical-to-acoustic energy conversion is linear. It is remarkable that this linear relationship occurs across the full immersion length range, even at the early stages of immersion (close to $d=0^{+}$), where the generation of sound comes from a much (small) localized fluid 
Publishingume, and thus many other factors no directly related to fluid volume change (including surface tension) could have also affected the frequency shift.

In the liquid droplet experiments reported here the change in the probe's resonance frequency is negative because the surrounded water molecules, being in their bulk state, are compliant to follow the probe's lateral motion. We conjectured that a similar transfer of energy may happen in near field experiments when testing the response from confined mesoscopic fluids. In the latter case, however, the molecules in the fluid are not as compliant to move along with the probe since they are instead more tightly attached to the substrate (zero-slip condition effect). As a consequence there will be a restoring force on the probe, which would lead to an increase in the observed resonance frequency (instead of a negative one, like in bulk fluid).

\section{CONCLUSIONS}

We have addressed the interaction between a laterally oscillating cylindrical fiber-probe and a sizable (few $\mu$ ) volume of fluid. A quartz tuning fork (TF) sensor monitored the response from the probe, recording its variations in oscillation amplitude and resonance frequency; an acoustic sensor monitored the fluid's response.

The response signals were well described by a simple harmonic oscillator (SHO) model. The inertial mass model (tested using probes of different diameters and at different immersion lengths) predicted very well the observed resonance frequency red-shifts. The validation of this model provided a proper framework for the subsequent analysis of the experimental data. On one hand, the weak dependence of the damping component of the probe-fluid interaction on viscosity was attributed to the zero-slip hydrodynamic condition (i.e. weak role of sliding friction). On the other hand, a strong correlation was found between the probe's frequency shift and the acoustic signal generated by the fluid. Remarkably, this correlation occurred across the entire 0 to $280 \mu \mathrm{m}$ immersion length range. Further, the acoustic signal increased with the fluid's viscosity, which was explained also in terms of the zero-slip hydrodynamic condition: water molecules are forced to move with the solid boundary, with the viscosity helping to drag an additional mass of fluid (contained in a $\sim 5 \mu \mathrm{m}$ thick boundary layer surrounding the probe) and thus contributing to the acoustic signal.

We underscore the role played by the relatively new Near-field Scanning Acoustic NearField Microscopy (SANM) technique in these measurements. Although the monotonic decrease 
Publishimg he probe's resonance amplitude and monotonic decrease in the probe's resonance frequency with probe immersion length was expected, the availability of the simultaneously monitored acoustic signal (obtained with the help of the SANM apparatus) was significant. Indeed, in the process of trying to find correlations (or lack of it) among these three signals led us to identify the important role played by the zero-slip condition.

Placing a substrate very close to an oscillating probe (initially interacting only with bulk fluid) would certainly cause new probe-fluid-substrate (near-field) interaction mechanisms to be considered. Nonetheless, the accumulated experimental evidence from probe/bulk-fluid interactions about the role played by the zero-slip boundary condition (being responsible for dragging the fluid molecules contained in boundary layer surrounding the probe and thus generating sound) suggests that a similar dynamic mechanism could also be present in near-field probe-fluid-substrate interactions. But in the latter case we have to consider that those fluid molecules in the boundary layer will not be as compliant to move along the probe as in the bulk case, because they are now also affected by adhesion forces exerted by the nearby stationary substrate. As a result, the net effect is a probe experiencing instead a spring type restoring force with the consequent increase in the probe's resonance frequency. This proposed hypothesis to explain the blue-shift in the probe's resonance frequency in near-field probe-fluid-substrate interactions is supported by experimental data accounted when the SANM was first introduced. ${ }^{5}$ The reported correlation between the probe's frequency shift and the fluid's acoustic signal from those near-field experiments can indeed be understood by invoking the zero-slip condition that the confined fluid must fulfill at the probe's walls and at the substrate.

\section{REFERENCES}

1 K. Karrai and R. D. Grober, "Piezoelectric tip-sample distance control for near field optical microscopes," Appl. Phys. Lett. 66, 1842-1844 (1995).

F. J. Giessibl, "Atomic resolution on $\mathrm{Si}(111)-(7 \mathrm{x} 7)$ by noncontact atomic force microscopy with a force sensor based on a quartz tuning fork," Appl. Phys. Lett. 76, 1470-1472 (2000). 
3 A. Gil, J. Colchero, M. Luna, J. Gómez-Herrero and A. M. Baro, “Adsorption of Water on Solid Surfaces Studied by Scanning Force Microscopy,” Langmuir 16, 5086-5092 (2000).

4 B. Kim, S. Kwon, H. Mun, S. An and W. Jhe, "Energy dissipation of nanoconfined hydration layer: Long-range hydration on the hydrophilic solid surface," Scientific Reports 4, 6499 (2014).

5 A. La Rosa, X. Cui, J. McCollum, N. Li and R. Nordstrom, "The ultrasonic/shear-force microscope: Integrating ultrasonic sensing into a near-field scanning optical microscope," Rev. Sci. Instrum. 76, 093707 (2005).

6 A. H. La Rosa, N. Li, and K. Asante, "The ultrasonic/shear-force microscope: a metrology tool for surface science and technology," Invited Paper, in "Nanofabrication: Technologies, Devices, and Applications II," Warren Y. Lai, L. E. Ocola, Stanley Pau Eds., Symposium in Optics East 2005, Boston, MA. Proc. SPIE 6002, 163-170 (2005).

7 X. Cui and A. H. La Rosa, "Investigation of the probe-sample interaction in the ultrasonic/shear force microscope: The phononic friction mechanism," Appl. Phys. Lett. 87, 231907 (2005).

8 M. Ohtsu, "Near-field Nano/Atom Optics and Technology," Springer, Tokyo (1998).

9 J. B. Segur and H. E. Oberstar, "Viscosity of Glycerol and Its Aqueous Solutions," Ind. Eng. Chem 43, 2117-2120 (1951).

${ }^{10}$ C. S. Miner and N. N. Dạlton, Glycerol: American Chemical Society Monograph Series, No. 117, Publisher: Literary Licensing, LLC (2013).

11 A. H. La Rosa, N. Li, R. Fernandez, X. Wang, R. Nordstrom and S. K. Padigi, "Whisperinggallery acoustic sensing: Characterization of mesoscopic films and scanning probe microscopy applications, ” Rev. Sci. Instrum. 82, 093704 (2011).

12 J. Rychen, T. Ihn, P. Studerus, A. Herrmann and K. Ensslin, "Operation characteristics of piezoelectric quartz tuning forks in high magnetic fields at liquid helium temperatures," Rev. Sci. Instrum. 71, 1695-1697 (2000). 
${ }^{13}$ M. Lee, J. Jahng, K. Kim and W. Jhe, "Quantitative atomic force measurement with a quartz tuning fork," Appl. Phys. Lett. 91, 023117 (2007).

14 K. Karrai and R. D. Grober, "Near Field Optics,” in: M. A. Paesler and P. J. Moyer (Ed.), SPIE Proceedings Series Vol. 2535, Bellingham, WA, 1995, pp. 69-81 (1995).

15 M. Christen, "Air and gas damping of quartz tuning forks," Sensors and Actuators 4, 555-564 (1983).

16 J. E. Sader, "Frequency response of cantilever beams immersed in viscous fluids with applications to atomic force microscope," J. Appl. Phys. 84, 64-76 (1998).

17 W. T. Blake, "The radiation from free-free beams in air and water," J. Sound Vib. 33, 427-450 (1974).

${ }^{18}$ R. K. Jeyapalan and E. J. Richards, "Radiation efficiencies of beams in flexural vibration," J. Sound Vib. 67, 55-67 (1979).

19 A Castellanos-Gomez, N. Agraït and G Rubio-Bollinger, "Dynamics of quartz tuning fork force sensors used in scanning probe microscopy," Nanotechnology 20, 215502 (2009).

${ }^{20}$ R. Fernandez, X. Wang, and A. H. La Rosa, "Acousto Characterization of Fluid-like Mesoscopic Films under Shear," Proceedings of the Nanotechnology (IEEE-NANO) 11th IEEE International Conference, pp. 903-906 (2011).

${ }^{21}$ In light of the additional inertial mass model described in the text, and in order to isolate the contribution only due to the viscosity, we should factor out from the $25 \mathrm{~Hz}$ change the contribution due to the larger density $\rho_{50 \%} / \rho_{0 \%}=1.13$, which gives a $22 \mathrm{~Hz}$ value. This represents a $10 \%$ rate larger decrease in frequency shift per immersion length caused by a 500 $\%$ increase in viscosity.

22 D. Schaeffel, S. Yordanov, M. Schmelzeisen, T. Yamamoto, M. Kappl, R. Schmitz, B. Dunweg, H. Butt and K. Koynov, "Hydrodynamic boundary condition of water on hydrophobic surfaces," Phys. Rev. E 87, 051001(R) (2013).

${ }^{23}$ Q. Yuan, and Y. Zhao, "Multiscale dynamic wetting of a droplet on a lyophilic pillar-arrayed surface," J. Fluid Mech. 716, 171-188 (2013). 
${ }^{24}$ Q. Yuan, X. Huang and Y. Zhao, "Dynamic spreading on pillar-arrayed surfaces: Viscous resistance versus molecular friction," Phys. of Fluids 26, 092104 (2014).

${ }^{25}$ M. A. Drummond Roby, G. C. Wetsel Jr. and C.-Y. Wang, “Scanned-probe lateral-force determination of fluid-dynamic effects near a solid surface in air," Appl. Phys. Lett. 69, 130 (1996).

${ }^{26}$ U. Raviv, P. Laurat and J. Klein, "Fluidity of water confined to subnanometer films," Nature 413, 51 (2001).

${ }^{27}$ S. H. Khan, G. Matei, S. Patil and P. M. Hoffmann, 'Dynamic Solidification in Nanoconfined Water Films,” Phys. Rev. Lett. 105, 106101 (2010).

${ }^{28}$ K. Karrai and I. Tiemann, "Interfacial shear force microscopy," Physical Review B 62, 1317413181 (2000).

${ }^{29}$ L. D. Landau and E. M. Lifshitz, Fluid Mechanics, $2^{\text {nd }}$ Edition, Pergamon Press (1987). See section 24 . 


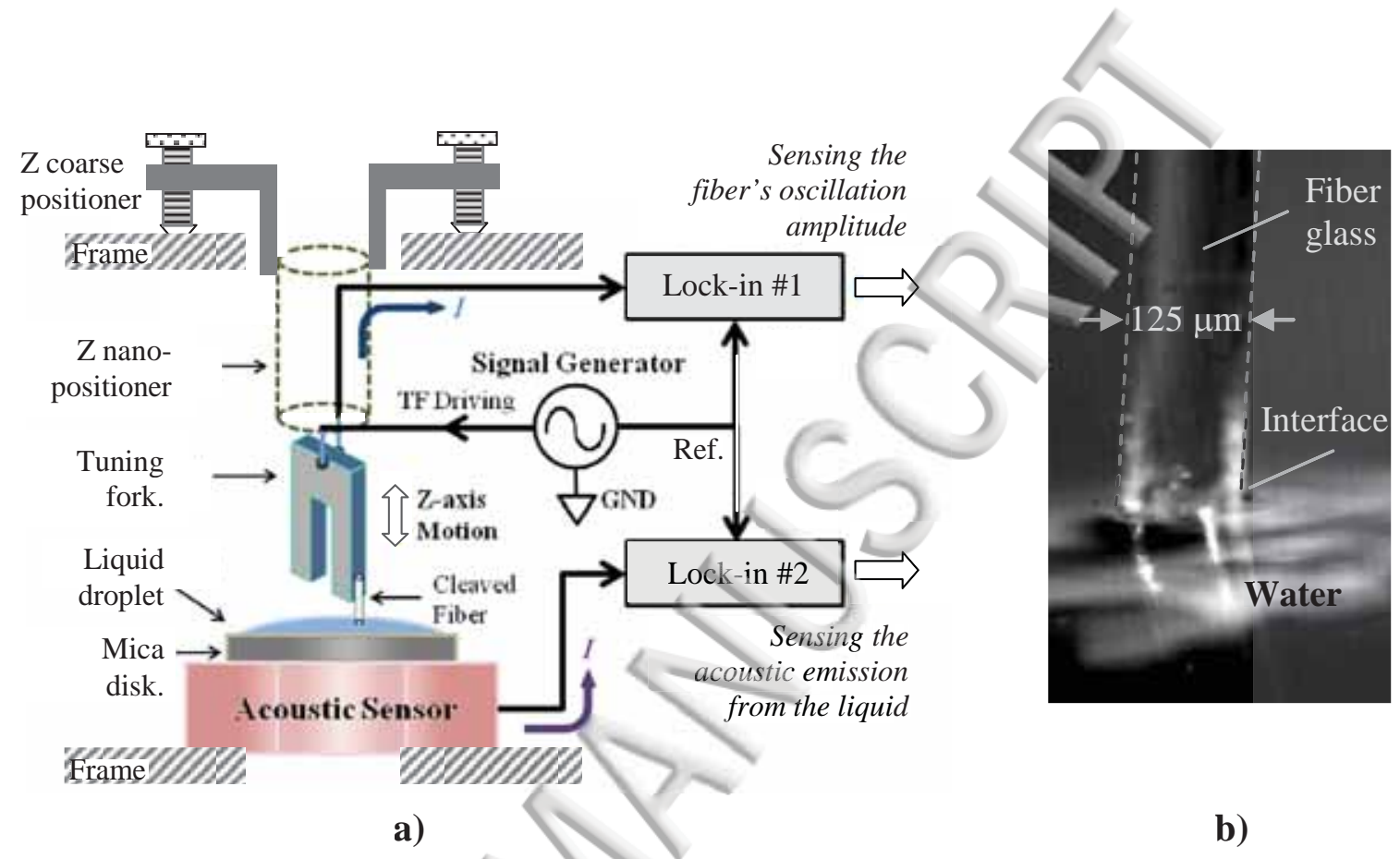

Fig. 1 
(a)

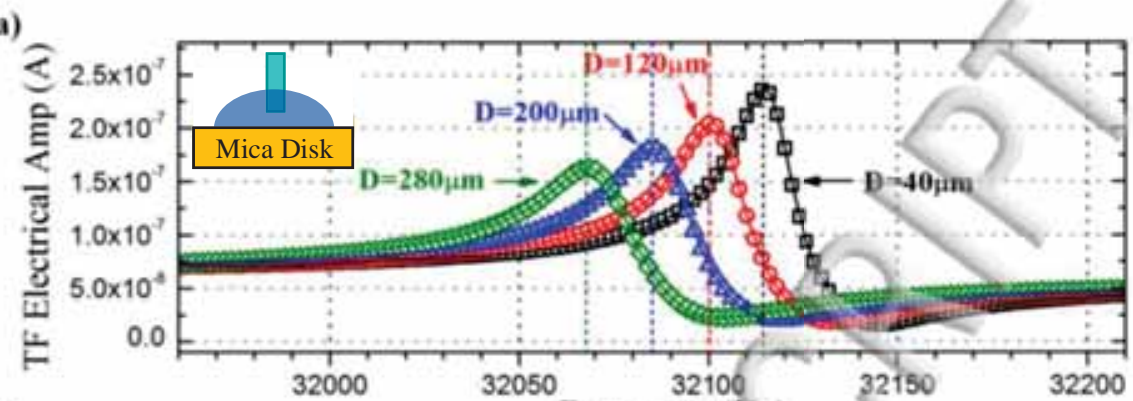

(b)

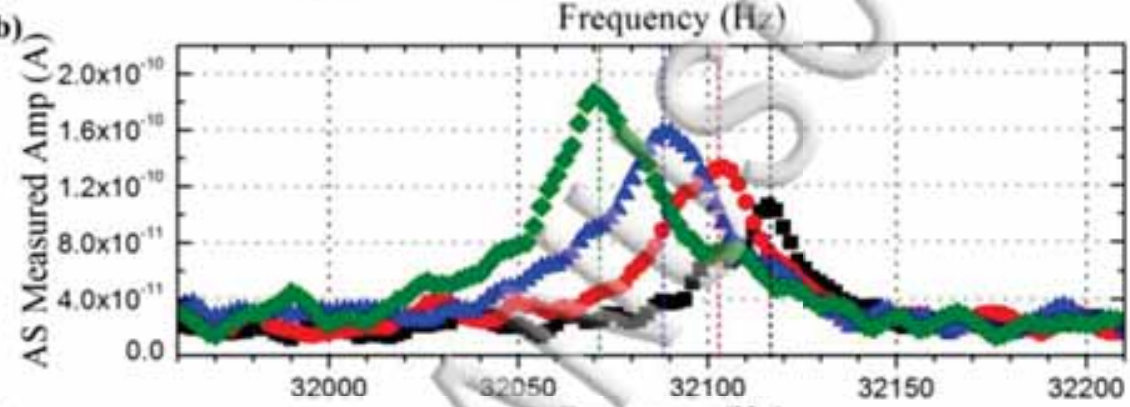

(c)

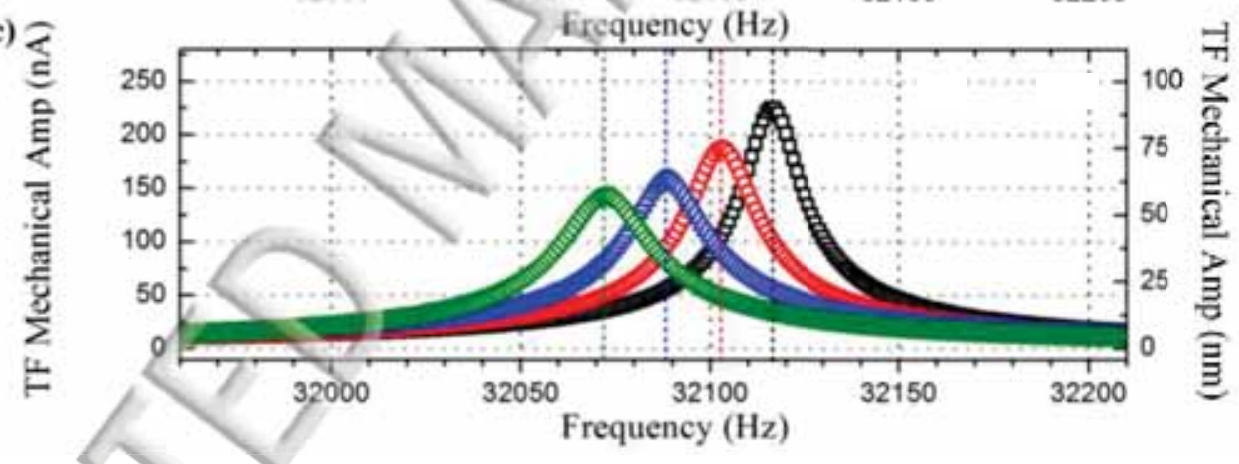

Fig. 2. 

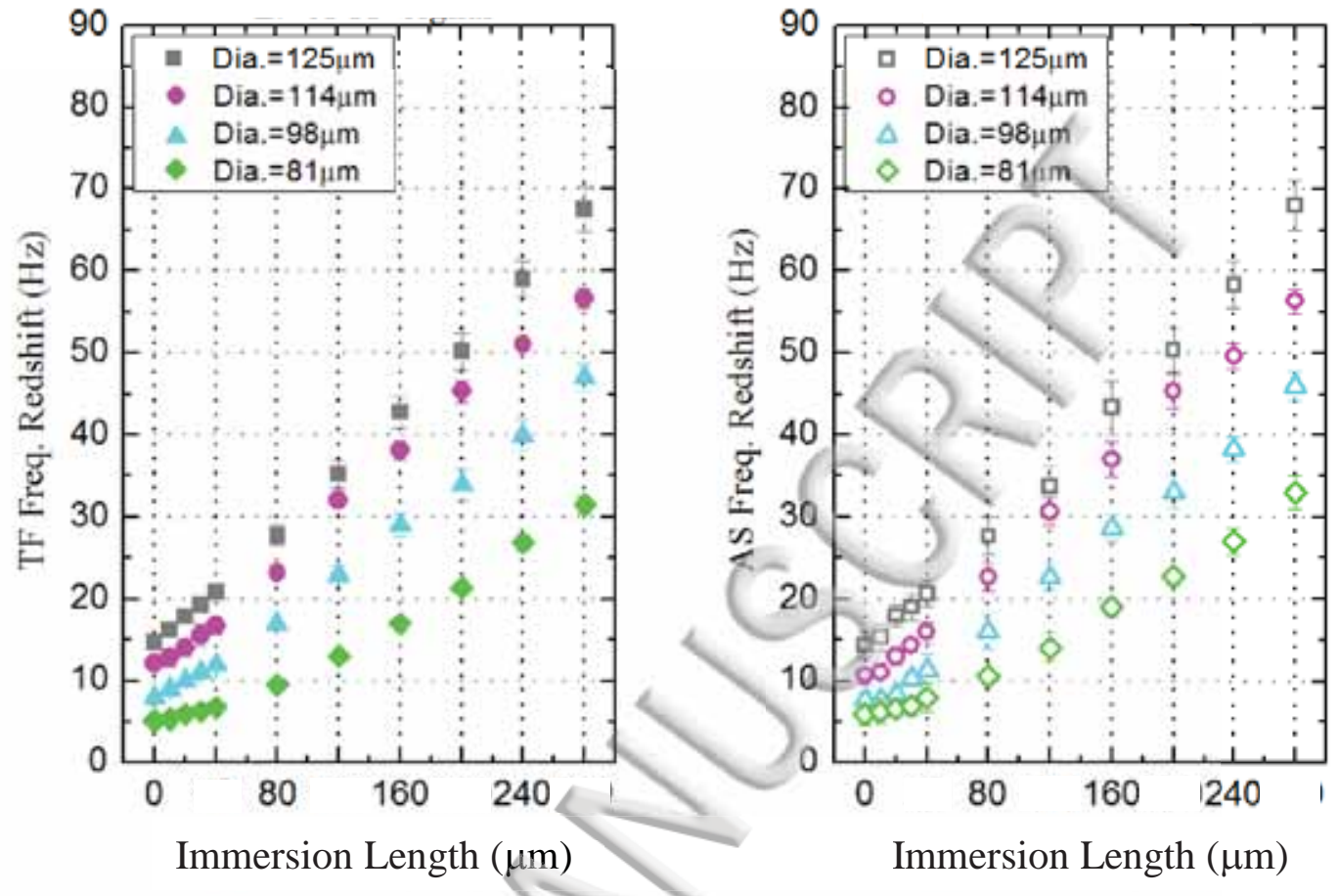

Fig. 3 

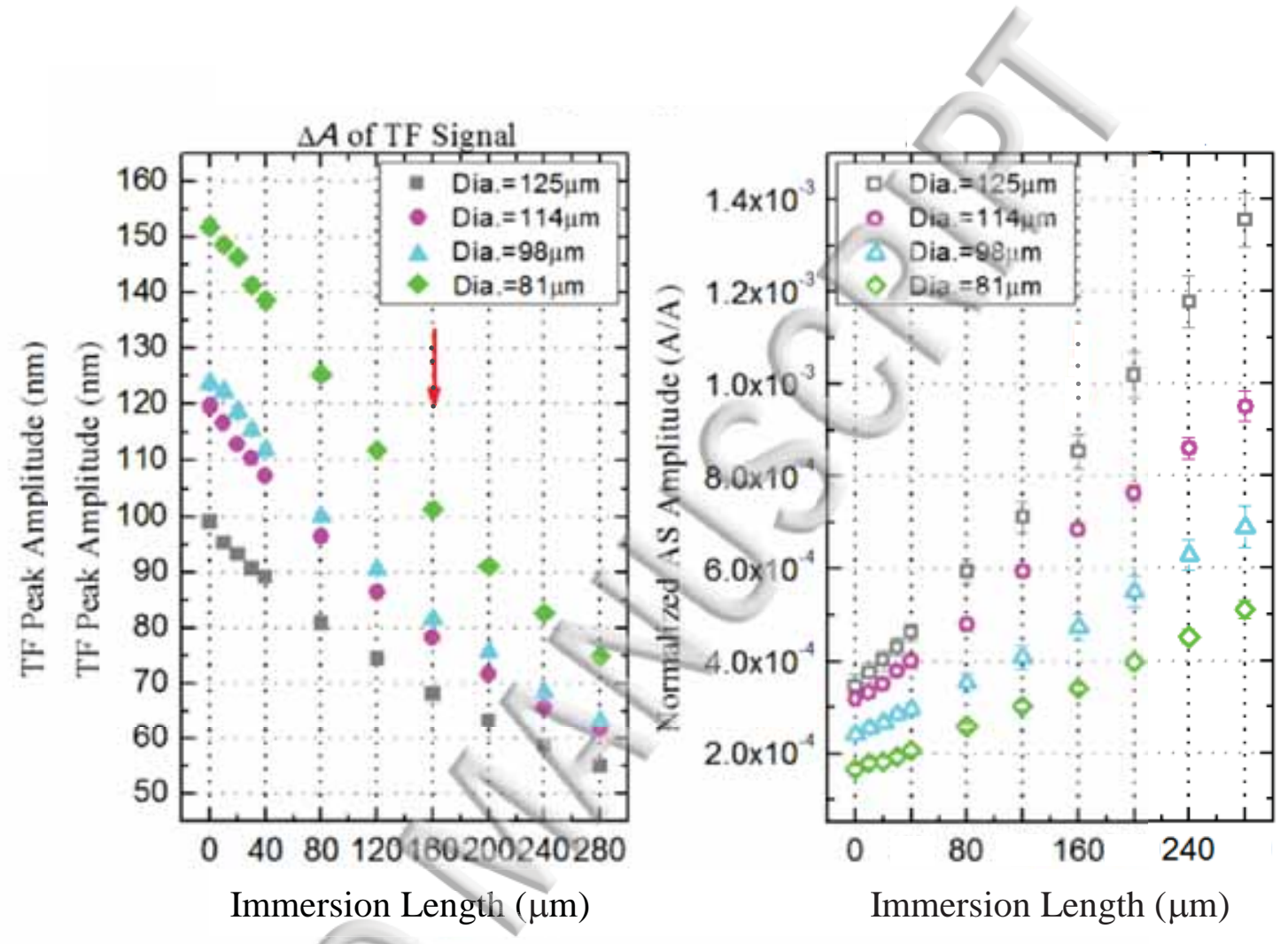

Fig. 4 

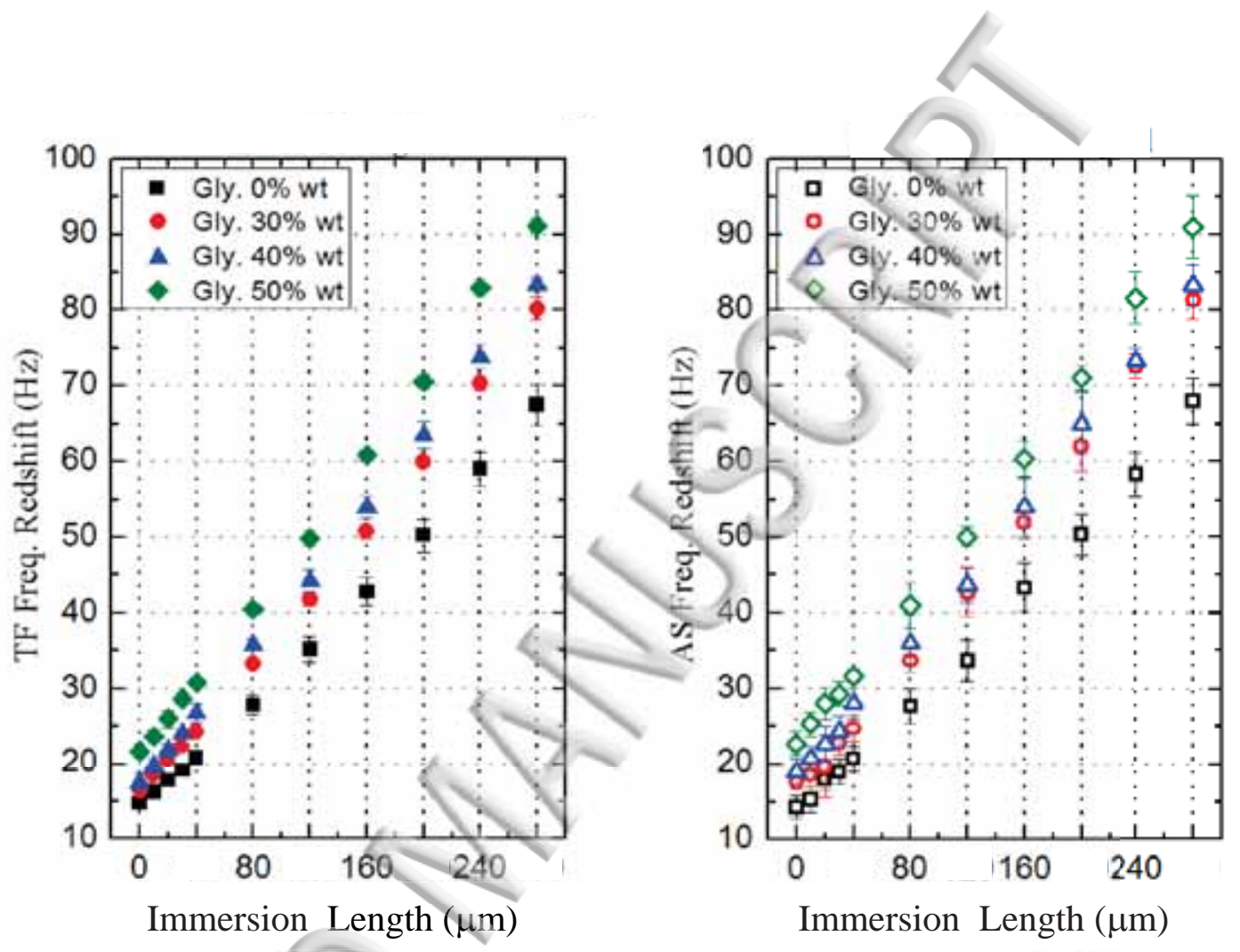

Fig. 5 

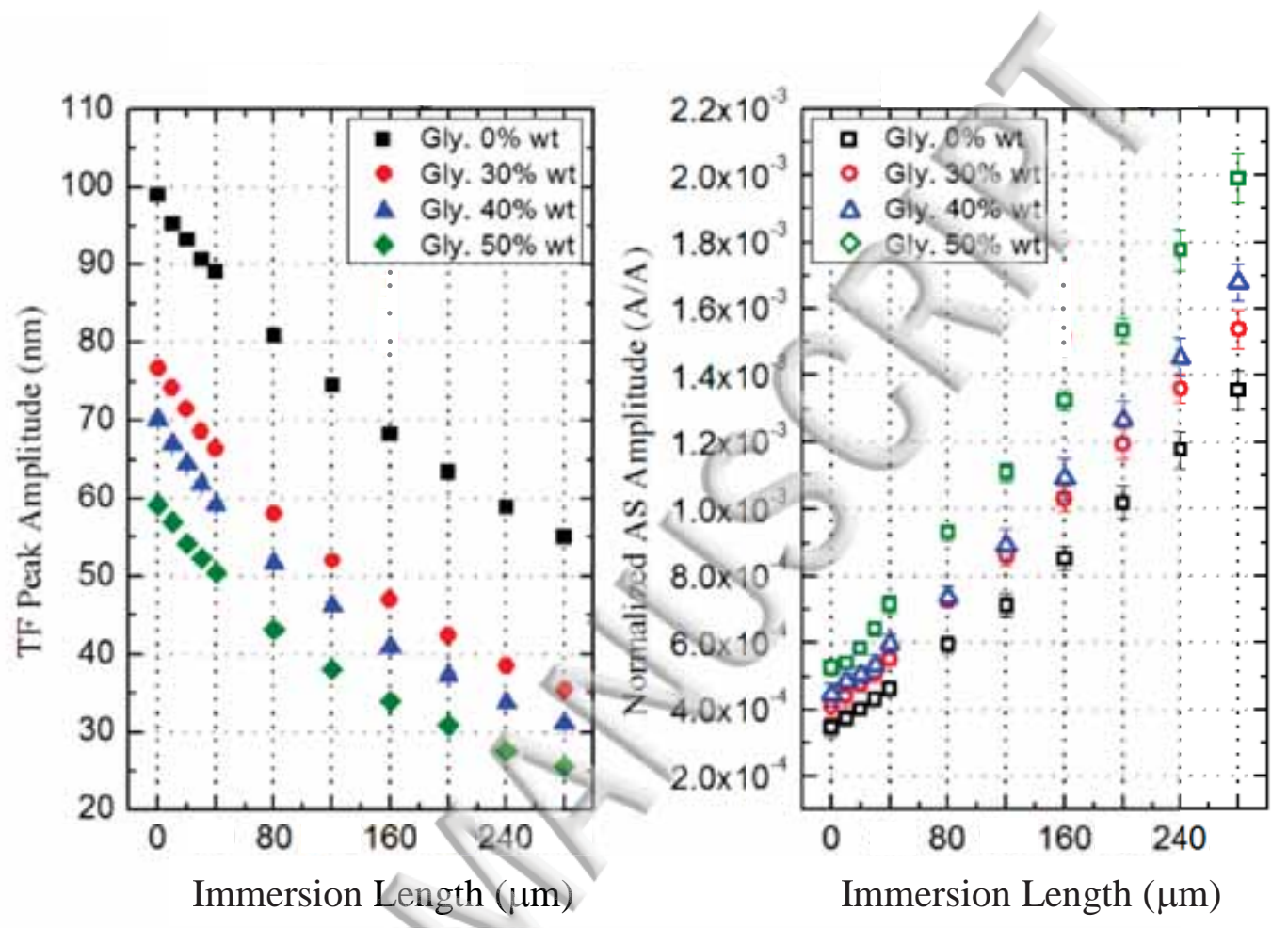

Fig. 6 

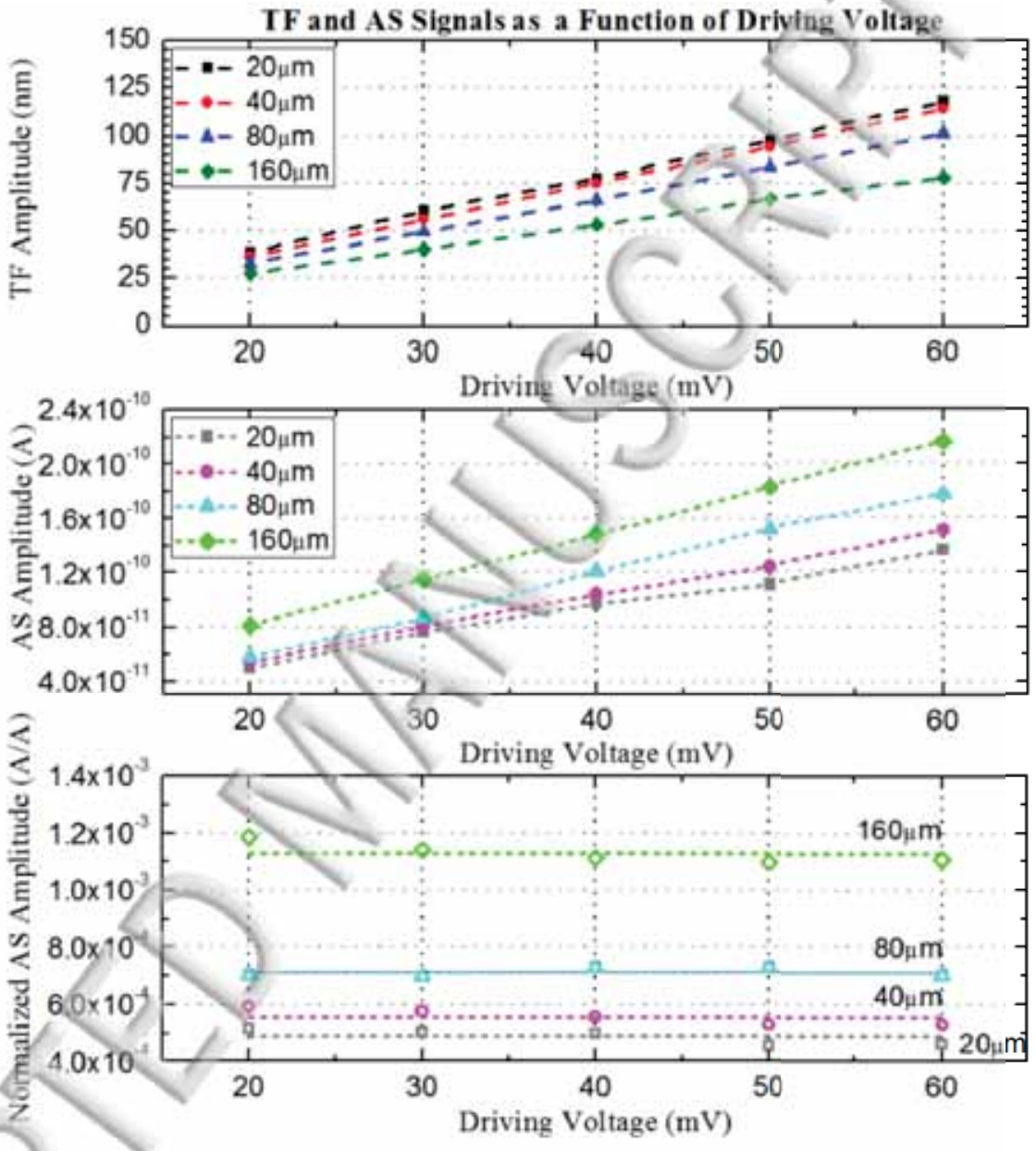

Fig. 7 


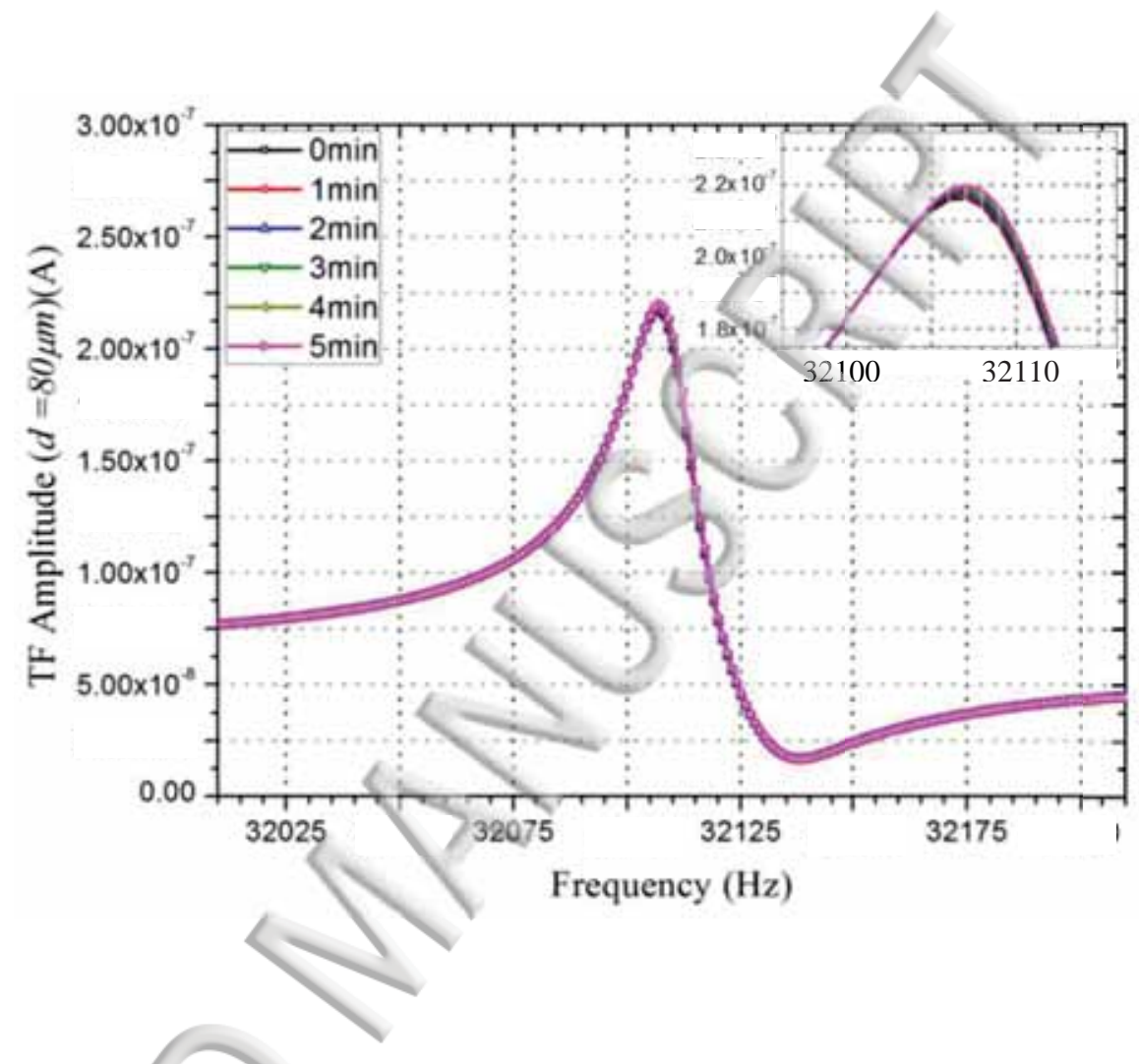

Fig. 8 
TF and AS Signals at Depth $=80 \mu \mathrm{m}$ in Drops with Different Volume

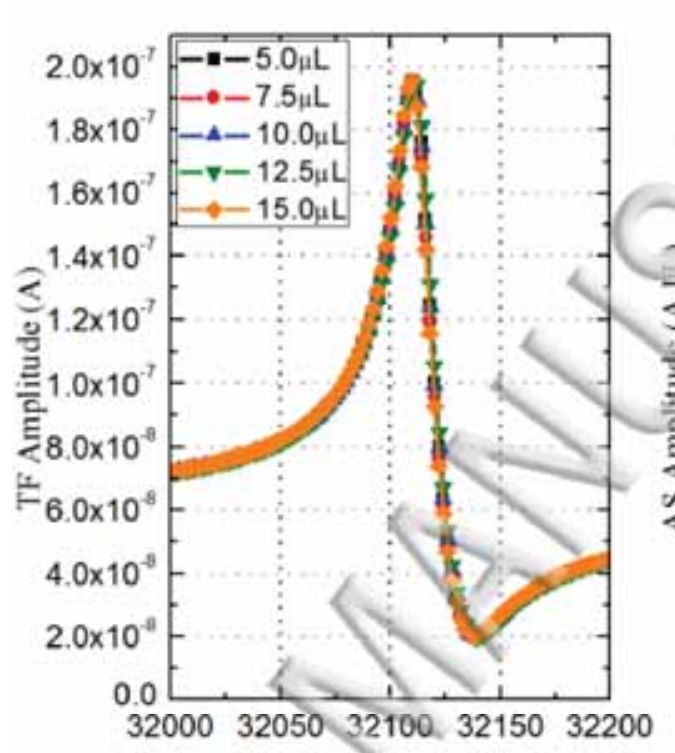

Frequency $(\mathrm{Hz})$

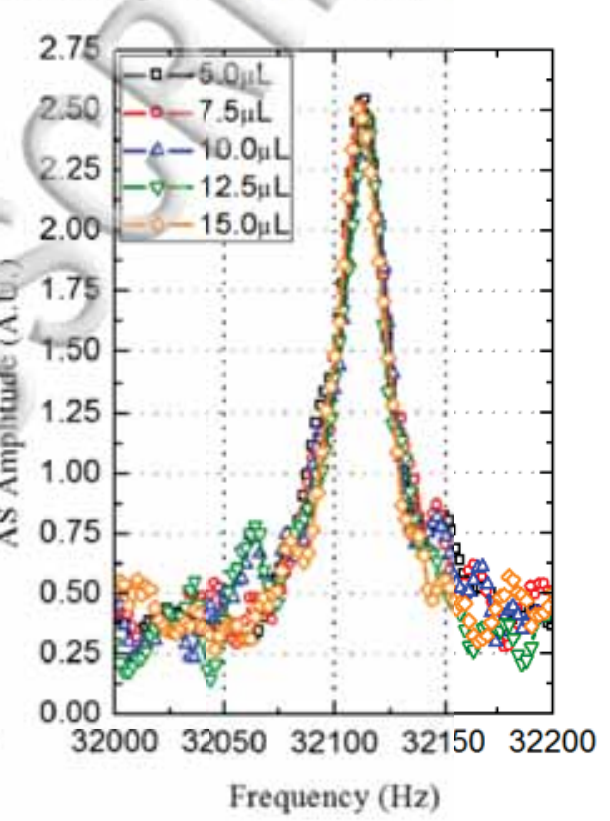

Fig. 9 


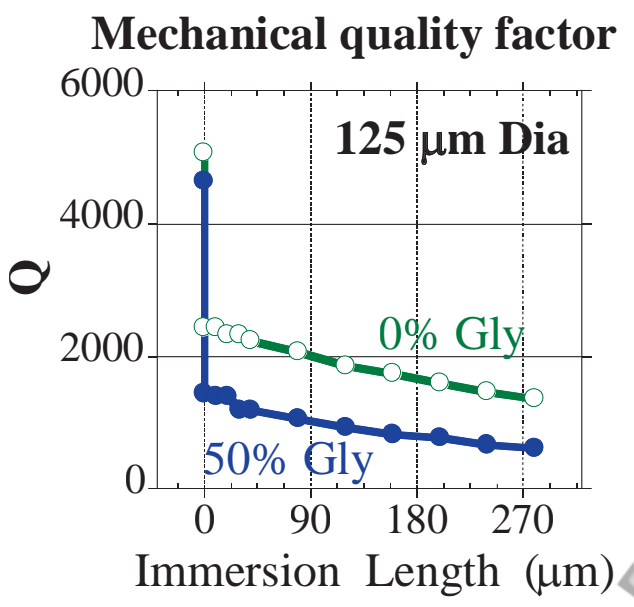

a)

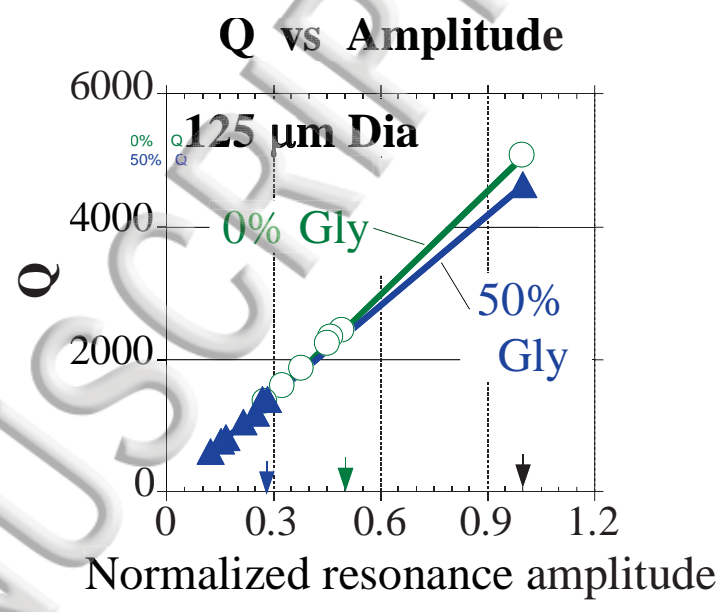

b)

Fig. 10 


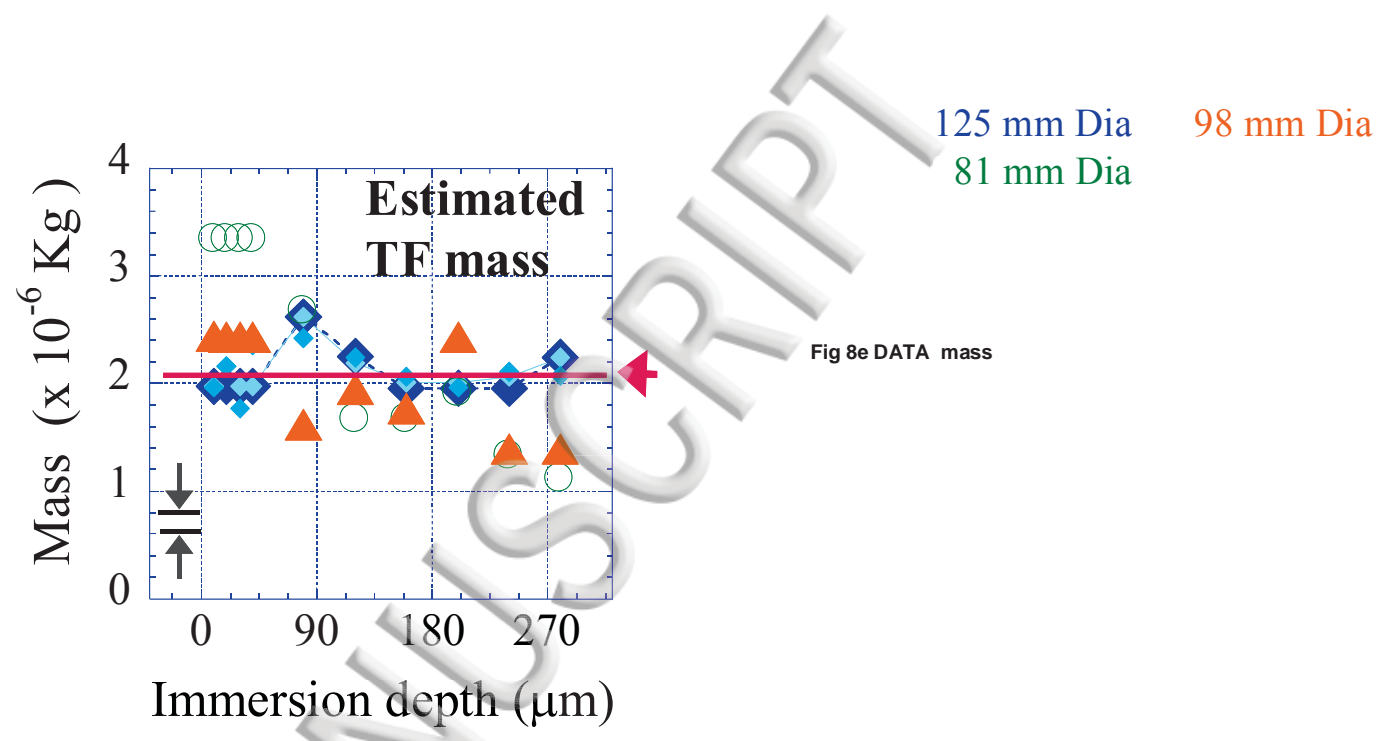

Fig 11 


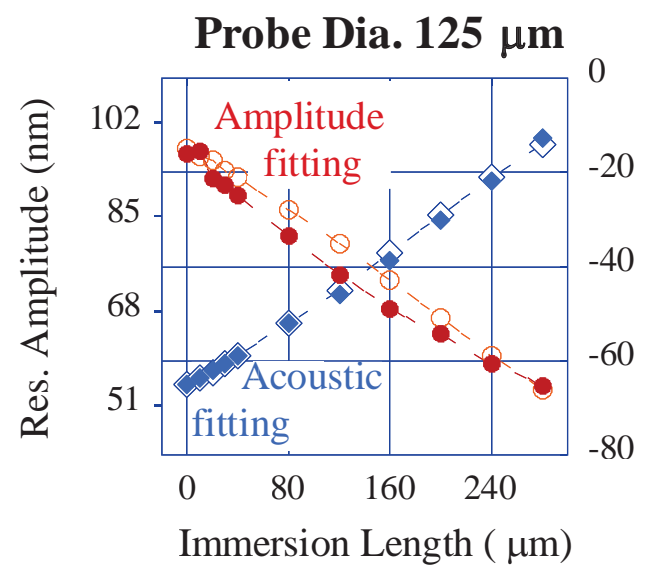

a)

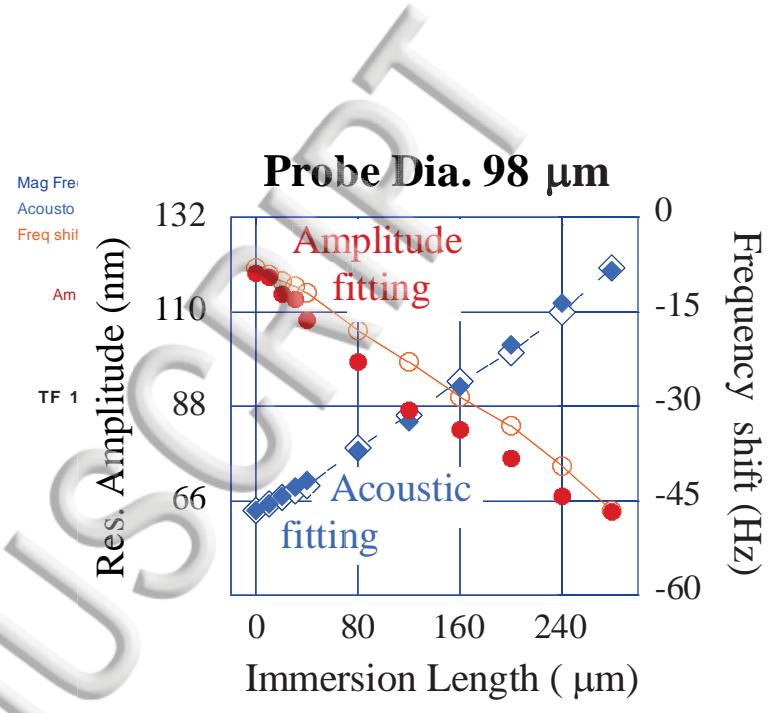

b)

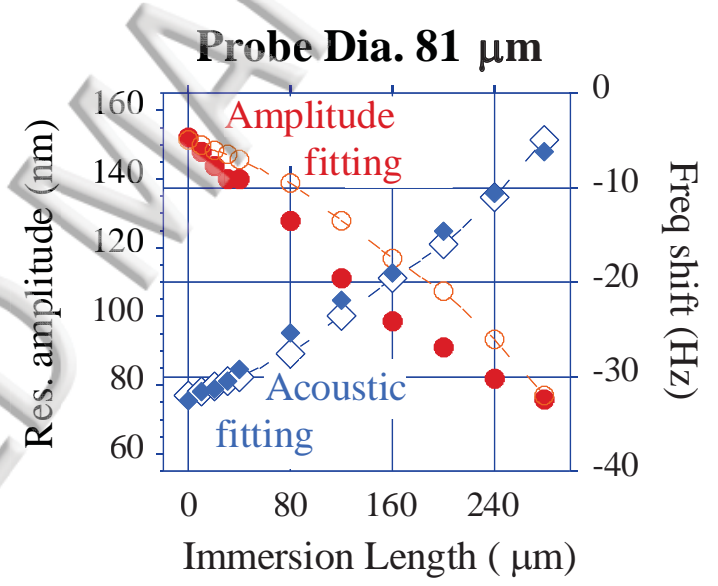

c)

Fig. 12 